Article

\title{
The Existence and Uniqueness of an Entropy Solution to Unilateral Orlicz Anisotropic Equations in an Unbounded Domain
}

\author{
Omar Benslimane ${ }^{1, *,+}\left(\mathbb{D}\right.$, Ahmed Aberqi ${ }^{2,+} \mathbb{C}$ and Jaouad Bennouna ${ }^{1,+}$ \\ 1 Laboratory LAMA, Department of Mathematics, Faculty of Sciences Dhar El Mahraz, B.P 1796 Atlas, \\ Sidi Mohamed Ben Abdellah University, Fez 30050, Morocco; jbennouna@hotmail.com \\ 2 Laboratory LAMA, National School of Applied Sciences, Sidi Mohamed Ben Abdellah University, \\ Fez 30050, Morocco; aberqi_ahmed@yahoo.fr \\ * Correspondence: omar.benslimane@usmba.ac.ma \\ + These authors contributed equally to this work.
}

Received: 5 July 2020; Accepted: 12 September 2020; Published: 17 September 2020

check for updates

\begin{abstract}
The purpose of this work is to prove the existence and uniqueness of a class of nonlinear unilateral elliptic problem $(\mathcal{P})$ in an arbitrary domain, managed by a low-order term and non-polynomial growth described by an $N$-uplet of $N$-function satisfying the $\Delta_{2}$-condition. The source term is merely integrable.
\end{abstract}

Keywords: anisotropic elliptic equation; obstacle problem; entropy solution; Sobolev-Orlicz anisotropic spaces; unbounded domain; existence and uniqueness

MSC: 35J47; 35J60

\section{Introduction}

Let $\Omega$ be an arbitrary domain of $\mathbb{R}^{N},(N \geq 2)$. In this paper, we investigate the existence and uniqueness solution of the following problem:

$$
(\mathcal{P}) \begin{cases}A(u)+\sum_{i=1}^{N} b_{i}(x, u, \nabla u)=f & \text { in } \Omega, \\ u \geq \psi & \text { a.e. in } \Omega,\end{cases}
$$

where, $A(u)=\sum_{i=1}^{N}\left(a_{i}(x, u, \nabla u)\right)_{x_{i}}$ is a Leray-Lions operator defined on $\mathrm{W}_{B}^{1}(\Omega)$ (defined as the adherence space $\left.C_{0}^{\infty}(\Omega)\right)$ into its dual; $B(t)=\left(B_{1}(t), \cdots, B_{N}(t)\right)$ are $N$-uplet Orlicz functions that satisfy $\Delta_{2}$-condition; the obstacle $\psi$ is a measurable function that belongs to $L^{\infty}(\Omega) \cap W_{B}^{1}(\Omega)$; and for $i=1, \cdots, N, b_{i}(x, s, \xi): \Omega \times \mathbb{R} \times \mathbb{R}^{N} \longrightarrow \mathbb{R}$ are Carathéodory functions (measurable with respect to $x$ in $\Omega$ for every $(s, \xi)$ in $\mathbb{R} \times \mathbb{R}^{N}$, and continuous with respect to $(s, \xi)$ in $\mathbb{R} \times \mathbb{R}^{N}$ for almost every $x$ in $\Omega$ ) that do not satisfy any sign condition and the growth described by the vector $N$-function $B(t)$. Take $f \in L^{1}(\Omega)$ too.

Statement of the problems: Suppose they have non-negative measurable functions $\phi, \varphi \in L^{1}(\Omega)$; and $\bar{a}, \tilde{a}$ are two constants, positive, such that for $\xi=\left(\xi_{1}, \cdots, \xi_{N}\right) \in \mathbb{R}^{N}$ and $\xi^{\prime}=\left(\xi_{1}^{\prime}, \cdots, \xi_{N}^{\prime}\right) \in \mathbb{R}^{N}$, we have

$$
\sum_{i=1}^{N}\left[a_{i}(x, s, \xi)-a_{i}\left(x, s, \xi^{\prime}\right)\right] \cdot\left(\xi_{i}-\xi_{i}^{\prime}\right)>0,
$$




$$
\begin{gathered}
\sum_{i=1}^{N} a_{i}(x, s, \xi) . \xi_{i} \geq \bar{a} \sum_{i=1}^{N} B_{i}\left(\left|\xi_{i}\right|\right)-\phi(x), \\
\sum_{i=1}^{N}\left|a_{i}(x, s, \xi)\right| \leq \tilde{a} \sum_{i=1}^{N} \bar{B}_{i}^{-1} B_{i}(|\xi|)+\varphi(x),
\end{gathered}
$$

and

$$
\sum_{i=1}^{N}\left|b_{i}(x, s, \xi)\right| \leq h(x)+l(s) \cdot \sum_{i=1}^{N} B_{i}(|\xi|),
$$

with $\bar{B}(t)$ being the complementary function of $B(t), h \in L^{1}(\Omega)$ and $l: \mathbb{R} \longrightarrow \mathbb{R}^{+}$being a positive continuous function such that $l \in L^{1}(\Omega) \cap L^{\infty}(\Omega)$.

We recall that in the last few decades, tremendous popularity has been achieved by the investigation of a class of nonlinear unilateral elliptic problem due to their fundamental role in describing several phenomena, such as the study of fluid filtration in porous media, constrained heating, elastoplasticity, optimal control, financial mathematics and others; for those studies, there are large numbers of mathematical articles; see [1-4] for more details.

When $\Omega$ is a bounded open set of $\mathbb{R}^{N}$, we refer to the celebrated paper by Bénilan [5], who presented the idea of entropy solutions adjusted to Boltzmann conditions. For more outcomes concerning the existence of solutions of this class in the Lebesgue Sobolev spaces (to be specific $\left.B(t)=|t|^{p}\right)$, we cite $[6,7]$. We cite $[4,8,9]$ for the Sobolev space with variable exponent. In the case of Orlicz spaces, we have some difficulties due to the non-homogeneity of the $N$-functions $B(t)$ and a rather indirect definition of the norm. It is generally difficult to move essentially $L^{p}$ techniques to Orlicz spaces. For more work within this framework, we quote [10-13].

On the other hand, when $\Omega$ is an unbounded domain, namely, without expecting any assumptions on the behavior when $|x| \longrightarrow+\infty$, Domanska in [14] investigated the well-posedness of nonlinear elliptic systems of equations generalizing the model equation

$$
-\sum_{i=1}^{N}\left(\left|u_{x_{i}}(x)\right|^{p_{i}-2} u_{x_{i}}(x)\right)_{x_{i}}+|u(x)|^{p_{0}-2} u(x)=f(x),
$$

with corresponding indices of nonlinearity $p_{i}>1(i=\overline{0, n})$. In [15] Bendahmann et al. the problem $(\mathcal{P})$ with $b(x, u, \nabla u)=\operatorname{div}(g(u))$ and $g(u)$ a polynomial growth like $u^{q}$ in $L^{p}$-spaces was solved. For more results we refer the reader to the work [16]. We mention [17-19], for the Sobolev space with variable exponent, and [20-26] for the classical anisotropic space.

The oddity of our present paper is to continue in this direction and to show the existence and uniqueness of entropy solution for equations $(\mathcal{P})$ governed with growth and described by an $N$-uplet of $N$-functions satisfying the $\Delta_{2}$-condition, within the fulfilling of anisotropic Orlicz spaces. Besides, we address the challenges that come about due to the absence of some topological properties, such as the densities of bounded or smooth functions.

The outline of this work is as follows. In Section 2, we recall some definitions and properties of $\mathrm{N}$-functions and the space of Sobolev-Orlicz anisotropic solutions. In Section 3, we prove the Theorem of the existence of the solutions in an unbounded domain with the help of some propositions; to be demonstrated later. In Section 4, we show the uniqueness of the solution to this problem, which is expected for strictly monotonic operators at least for a broad class of lower-order terms. Finally, there is Appendix A.

\section{Mathematical Background and Auxiliary Results}

In this section, we introduce the notation, recall some standard definitions and collect necessary propositions and facts that are used to establish our main result.

A comprehensive presentation of Sobolev-Orlicz anisotropic space can be found in the books of M.A Krasnoselskii and Ja. B. Rutickii [23] and in [20,25]. 
Definition 1. We say that $B: \mathbb{R}^{+} \longrightarrow \mathbb{R}^{+}$is a $N$-function if $B$ is continuous, convex, with $B(z)>0$ for $z>0$, $\frac{B(z)}{z} \rightarrow 0$ when $z \rightarrow 0$ and $\frac{B(z)}{z} \rightarrow \infty$ when $z \rightarrow \infty$.

This N-function $B$ admits the following representation: $B(z)=\int_{0}^{z} b(t) d t$, with $b: \mathbb{R}^{+} \longrightarrow \mathbb{R}^{+}$which is an increasing function on the right, with $b(0)=0$ in the case $z>0$ and $b(z) \longrightarrow \infty$ when $z \longrightarrow \infty$.

Its conjugate is noted by $\bar{B}(z)=\int_{0}^{|z|} q(t) d t$ with $q$ also satisfying all the properties already quoted from $b$, with

$$
\bar{B}(z)=\sup _{y \geq 0}(y|z|-B(y)), \quad z \in \mathbb{R} .
$$

The Young's inequality is given as follows:

$$
\forall z, y \in \mathbb{R} \quad|z y| \leq B(y)+\bar{B}(z)
$$

Definition 2. The $N$-function $B(z)$ satisfies the $\Delta_{2}$-condition if $\exists c>0, z_{0} \geq 0$ such that

$$
B(2 z) \leq c B(z) \quad|z| \geq z_{0}
$$

This definition is equivalent to, $\forall k>1, \exists c(k)>0$ such that

$$
B(k z) \leq c(k) B(z) \quad \text { for } \quad|z| \geq z_{0}
$$

Definition 3. The $N$-function $B(z)$ satisfies the $\Delta_{2}$-condition as long as there exist positive numbers $c>1$ and $z_{0} \geq 0$ such that for $|z| \geq z_{0}$ we have

$$
z b(z) \leq c B(z)
$$

Additionally, each $N$-function $B(z)$ satisfies the inequality

$$
B(y+z) \leq c B(z)+c B(y) \quad z, y \in \mathbb{R} \text {. }
$$

Definition 4. The $N$-function $B(z)$ satisfies the $\nabla_{2}$-condition if $\exists c>2, z_{0} \geq 0$ such that

$$
B(2 z) \leq c B(z) \quad|z| \geq z_{0}
$$

We consider the Orlicz space $L_{B}(\Omega)$ provided with the norm of Luxemburg given by

$$
\|u\|_{B, \Omega}=\inf \left\{k>0 / \int_{\Omega} B\left(\frac{u(x)}{k}\right) d x \leq 1\right\} .
$$

According with [23] we obtain the inequalities

$$
\int_{\Omega} B\left(\frac{u(x)}{\|u\|_{B, \Omega}}\right) d x \leq 1
$$

and

$$
\|u\|_{B, \Omega} \leq \int_{\Omega} B(u) d x+1 .
$$

Moreover, the Hölder's inequality holds and we have for all $u \in L_{B}(\Omega)$ and $v \in L_{\bar{B}}(\Omega)$

$$
\left|\int_{\Omega} u(x) v(x) d x\right| \leq 2\|u\|_{B, \Omega} \cdot\|v\|_{\bar{B}, \Omega} .
$$


In $[23,25]$, if $P(z)$ and $B(z)$ are two $N$-functions such that $P(z) \ll B(z)$ and meas $\Omega<\infty$, then $L_{B}(\Omega) \subset L_{P}(\Omega)$; furthermore,

$$
\|u\|_{P, \Omega} \leq A_{0}(\text { meas } \Omega)\|u\|_{B, \Omega} \quad u \in L_{B}(\Omega) .
$$

Additionally, for all $N$-functions $B(z)$, if meas $\Omega<\infty$, then $L_{\infty}(\Omega) \subset L_{B}(\Omega)$ with

$$
\|u\|_{B, \Omega} \leq A_{1}(\text { meas } \Omega)\|u\|_{\infty, \Omega} \quad u \in L_{B}(\Omega) .
$$

Additionally, for all $N$-functions $B(z)$, if meas $\Omega<\infty$, then $L_{B}(\Omega) \subset L^{1}(\Omega)$ with

$$
\|u\|_{1, \Omega} \leq A_{2}\|u\|_{B, \Omega} \quad u \in L_{B}(\Omega) .
$$

We define for all $N$-functions $B_{1}(z), \cdots, B_{N}(z)$ the space of Sobolev-Orlicz anisotropic $W_{B}^{1}(\Omega)$ as the adherence space $C_{0}^{\infty}(\Omega)$ under the norm

$$
\|u\|_{\stackrel{\mathrm{W}}{B}_{B}^{1}(\Omega)}=\sum_{i=1}^{N}\left\|u_{x_{i}}\right\|_{B_{i}, \Omega}
$$

Definition 5. A sequence $\left\{u_{m}\right\}$ is said to converge modularly to $u$ in $\mathrm{W}_{B}^{1}(\Omega)$ if for some $k>0$ we have

$$
\int_{\Omega} B\left(\frac{u_{m}-u}{k}\right) d x \longrightarrow 0 \text { as } m \longrightarrow \infty
$$

Remark 1. Since B satisfies the $\Delta_{2}$-condition, the modular convergence coincides with the norm convergence.

Remark 2. If the doubling condition is imposed on the modular function, but not on the conjugate, then the space for the solutions to exist is non-reflexive in general. For this reason we will assume in the remainder of this article that $B$ satisfies the both conditions; the $\Delta_{2}$-condition and $\nabla_{2}$-condition, so the Propositions 1 and 2 will remain true.

Proposition 1 ([23]). The Sobolev-Orlicz anisotropic space $\mathrm{W}_{B}^{1}(\Omega)$ is complete and reflexive.

Proposition 2 ([23]). The Sobolev-Orlicz anisotropic $\mathrm{W}_{B}^{1}(\Omega)$ is separable.

\section{Proposition 3.}

$$
z B^{\prime}(z)=\bar{B}\left(B^{\prime}(z)\right)+B(z), \quad z>0,
$$

with $B^{\prime}$ being the right derivative of the $N$-function $B(z)$.

Proof. By (6), we take $y=B^{\prime}(z)$; then we obtain

$$
B^{\prime}(z) z \leq B(z)+\bar{B}\left(B^{\prime}(z)\right)
$$

and by Ch. I [23], we get the result.

In the following we will assume that for each $N$-function $B_{i}(z)=\int_{0}^{|z|} b_{i}(t) d t$ obeys the further condition

$$
\lim _{\alpha \rightarrow \infty} \inf _{\theta>0} \frac{b_{i}(\alpha \theta)}{b_{i}(\alpha)}=\infty, \quad i=1, \cdots, N .
$$

Example 1. The function

$$
B(z)=|z|^{b}(|\ln | z||+1),
$$


with $b>1$ checks the $\Delta_{2}$-condition and (22).

Lemma 1. Suppose that $(X, \mathcal{T}$, meas $)$ is a measurable set such that mes $(X)<\infty$. Let $\theta: X \longrightarrow[0,+\infty]$ be a measurable function such that meas $\{x \in X: \theta(x)=0\}=0$. Then, for any $\epsilon>0$, there exists $\delta>0$ such that for any bounded domain $Q$

$$
\int_{Q} \theta(x) d x \leq \delta
$$

implies that

$$
\operatorname{meas}(Q) \leq \epsilon
$$

Proof. See [27] (Lemma 2).

\section{The Existence of an Entropy Solution}

This section is devoted to the proofs of our main results which will be split into different steps. For $m \in \mathbb{N}^{*}$, we define the truncation at height $m, T_{m}(u): \mathbb{R} \longrightarrow \mathbb{R}$ by

$$
T_{m}(u)= \begin{cases}u & \text { if }|u| \leq m \\ m & \text { if }|u|>m\end{cases}
$$

Definition 6. A measurable function $u$ is said to be an entropy solution for the problem $(\mathcal{P})$, if $u \in \mathfrak{W}_{B}^{1}(\Omega)$ such that $u \geq \psi$ a.e. in $\Omega$ and

$$
\begin{aligned}
& \sum_{i=1}^{N} \int_{\Omega} a_{i}(x, u, \nabla u) \cdot \nabla(u-v) d x+\sum_{i=1}^{N} \int_{\Omega} b_{i}(x, u, \nabla u) \cdot(u-v) d x \\
& \quad+\int_{\Omega} m \cdot T_{m}(u-\psi)^{-} \cdot s g_{\frac{1}{m}}(u) \cdot(u-v) d x \\
& \quad \leq \int_{\Omega} f(x) \cdot(u-v) d x \quad \forall v \in K_{\psi} \cap L^{\infty}(\Omega),
\end{aligned}
$$

where, $K_{\psi}=\left\{u \in \stackrel{\circ}{B}_{B}^{1}(\Omega) / u \geq \psi\right.$ a.e. in $\left.\Omega\right\}$, and $s g_{m}(s)=\frac{T_{m}(s)}{m}$.

We have $f^{m} \longrightarrow f$ in $L^{1}(\Omega), m \rightarrow \infty,\left|f^{m}(x)\right| \leq|f(x)|$ and for $i=1, \cdots, N, a_{i}^{m}\left(x, u_{m}, \nabla u_{m}\right)$ : $\left(\stackrel{\circ}{B}_{B}^{1}(\Omega)\right)^{N} \longrightarrow\left(\stackrel{\circ}{W}_{\bar{B}}^{-1}(\Omega)\right)^{N}$ being Carathéodory functions with

$$
a_{i}^{m}(x, u, \nabla u)=a_{i}\left(x, T_{m}(u), \nabla u\right),
$$

and $b_{i}^{m}\left(x, u_{m}, \nabla u_{m}\right): \Omega \times \mathbb{R} \times \mathbb{R}^{N} \longrightarrow \mathbb{R}$ again being Carathéodory functions not satisfying any sign condition, with

$$
b^{m}(x, u, \nabla u)=\frac{b(x, u, \nabla u)}{1+\frac{1}{m}|b(x, u, \nabla u)|},
$$

and

$$
\left|b^{m}(x, u, \nabla u)\right|=\left|b\left(x, T_{m}(u), \nabla u\right)\right| \leq m \text { for all } m \in \mathbb{N}^{*},
$$

and for all $v \in \mathfrak{W}_{B}^{1}(\Omega)$, we consider the following approximate problem:

$$
\begin{aligned}
\left(\mathcal{P}_{m}\right): \sum_{i=1}^{N} & \int_{\Omega} a_{i}^{m}\left(x, u_{m}, \nabla u_{m}\right) \cdot \nabla\left(u_{m}-v\right) d x+\sum_{i=1}^{N} \int_{\Omega} b_{i}^{m}\left(x, u_{m}, \nabla u_{m}\right) \cdot\left(u_{m}-v\right) d x \\
& +\int_{\Omega} m \cdot T_{m}\left(u_{m}-\psi\right)^{-} \cdot \operatorname{sg}_{\frac{1}{m}}\left(u_{m}\right) \cdot\left(u_{m}-v\right) d x=\int_{\Omega} f^{m}(x) \cdot\left(u_{m}-v\right) d x
\end{aligned}
$$


Theorem 1. Assume that conditions (1)-(4) and (22) hold true, then there exists at least one solution of the approximate problem $\left(\mathcal{P}_{m}\right)$.

Proof. See Appendix A.

Theorem 2. Under assumptions (1)-(4), the problem $(\mathcal{P})$ has at least one entropy solution.

Proof. Let $R>0$ and $\Omega(R)=\{x \in \Omega:|x| \leq R\}$. Note by $h(t)=\left(\prod_{i=1}^{N} \frac{B_{i}^{-1}(t)}{t}\right)^{\frac{1}{N}}$ and we assume that $\int_{0}^{1} \frac{h(t)}{t} d t$ converge, so we consider the $N$-functions $B^{*}(z)$ defined by $\left(B^{*}\right)^{-1}(z)=\int_{0}^{|z|} \frac{h(t)}{t} d t$

Lemma 2 ([20]). Let $u \in \grave{W}_{B}^{1}(\Omega(R))$. If

$$
\int_{1}^{\infty} \frac{h(t)}{t} d t=\infty
$$

then $\stackrel{\circ}{B}_{B}^{1}(\Omega(R)) \subset L_{B^{*}}(\Omega(R))$ and $\|u\|_{B^{*}, \Omega(R)} \leq \frac{N-1}{N}\|u\|_{\grave{W}_{B}^{1}(\Omega(R))}$. If

$$
\int_{1}^{\infty} \frac{h(t)}{t} d t \leq \infty
$$

then $\stackrel{\circ}{B}_{B}^{1}(\Omega(R)) \subset L_{\infty}(\Omega(R))$ and $\|u\|_{\infty, \Omega(R)} \leq \beta\|u\|_{\stackrel{\circ}{B}_{B}^{1}(\Omega(R))}$, with $\beta=\int_{0}^{\infty} \frac{h(t)}{t} d t$.

Step 1. A priori estimate of $\left\{u_{m}\right\}$ :

Let $v=u_{m}-\eta \exp \left(G\left(u_{m}\right)\right) \cdot T_{k}\left(u_{m}-v_{0}\right)^{+}$where $G(s)=\int_{0}^{s} \frac{l(t)}{\bar{a}} d t, k>0$ and $\eta \geq 0$; we have $v \in \stackrel{W}{B}_{B}^{1}(\Omega)$ and for a small enough $\eta$ we deduce that $v \geq \psi$. Thus $v$ is an admissible test function in $\left(\mathcal{P}_{m}\right)$ and we get for all $v_{0} \in K_{\psi} \cap L^{\infty}(\Omega)$ that

$$
\begin{aligned}
& \sum_{i=1}^{N} \int_{\Omega} a_{i}^{m}\left(x, u_{m}, \nabla u_{m}\right) \cdot \nabla\left(\exp \left(G\left(u_{m}\right)\right) \cdot T_{k}\left(u_{m}-v_{0}\right)^{+}\right) d x \\
& \quad+\sum_{i=1}^{N} \int_{\Omega} b_{i}^{m}\left(x, u_{m}, \nabla u_{m}\right) \cdot \exp \left(G\left(u_{m}\right)\right) \cdot T_{k}\left(u_{m}-v_{0}\right)^{+} d x \\
& \quad+\int_{\Omega} m \cdot T_{m}\left(u_{m}-\psi\right)^{-} \cdot \operatorname{sg}_{\frac{1}{m}}\left(u_{m}\right) \cdot \exp \left(G\left(u_{m}\right)\right) \cdot T_{k}\left(u_{m}-v_{0}\right)^{+} d x \\
& \quad \leq \int_{\Omega} f^{m}(x) \cdot \exp \left(G\left(u_{m}\right)\right) \cdot T_{k}\left(u_{m}-v_{0}\right)^{+} d x
\end{aligned}
$$


then,

$$
\begin{aligned}
& \sum_{i=1}^{N} \int_{\Omega} a_{i}^{m}\left(x, u_{m}, \nabla u_{m}\right) \cdot \nabla u_{m} \cdot \frac{l\left(u_{m}\right)}{\bar{a}} \cdot \exp \left(G\left(u_{m}\right)\right) \cdot T_{k}\left(u_{m}-v_{0}\right)^{+} d x \\
& \quad+\sum_{i=1}^{N} \int_{\Omega} a_{i}^{m}\left(x, u_{m}, \nabla u_{m}\right) \cdot \exp \left(G\left(u_{m}\right)\right) \cdot \nabla T_{k}\left(u_{m}-v_{0}\right)^{+} d x \\
& \quad+\int_{\Omega} m \cdot T_{m}\left(u_{m}-\psi\right)^{-} \cdot \operatorname{sg}_{\frac{1}{m}}\left(u_{m}\right) \cdot \exp \left(G\left(u_{m}\right)\right) \cdot T_{k}\left(u_{m}-v_{0}\right)^{+} d x \\
& \quad \leq \sum_{i=1}^{N} \int_{\Omega}\left|b_{i}^{m}\left(x, u_{m}, \nabla u_{m}\right)\right| \cdot \exp \left(G\left(u_{m}\right)\right) \cdot T_{k}\left(u_{m}-v_{0}\right)^{+} d x \\
& \quad+\int_{\Omega} f^{m}(x) \cdot \exp \left(G\left(u_{m}\right)\right) \cdot T_{k}\left(u_{m}-v_{0}\right)^{+} d x,
\end{aligned}
$$

by (2) and (4), we obtain

$$
\begin{aligned}
& \sum_{i=1}^{N} \int_{\Omega} a_{i}^{m}\left(x, u_{m}, \nabla u_{m}\right) \cdot \exp \left(G\left(u_{m}\right)\right) \cdot \nabla T_{k}\left(u_{m}-v_{0}\right)^{+} d x \\
& \quad+\int_{\Omega} m \cdot T_{m}\left(u_{m}-\psi\right)^{-} \cdot \operatorname{sg}_{\frac{1}{m}}\left(u_{m}\right) \cdot \exp \left(G\left(u_{m}\right)\right) \cdot T_{k}\left(u_{m}-v_{0}\right)^{+} d x \\
& \quad \leq \int_{\Omega}\left[h(x)+f^{m}(x)+\phi(x) \cdot \frac{l\left(u_{m}\right)}{\bar{a}}\right] \cdot \exp \left(G\left(u_{m}\right)\right) \cdot T_{k}\left(u_{m}-v_{0}\right)^{+} d x
\end{aligned}
$$

so

$$
\begin{aligned}
& \sum_{i=1}^{N} \int_{\left\{\left|u_{m}-v_{0}\right| \leq k\right\}} a_{i}^{m}\left(x, u_{m}, \nabla u_{m}\right) \cdot \nabla u_{m} \cdot \exp \left(G\left(u_{m}\right)\right) d x \\
& \quad-c \sum_{i=1}^{N} \int_{\left\{\left|u_{m}-v_{0}\right| \leq k\right\}} a_{i}^{m}\left(x, u_{m}, \nabla u_{m}\right) \cdot \frac{\nabla v_{0}}{c} \cdot \exp \left(G\left(u_{m}\right)\right) d x \\
& \quad+\int_{\Omega} m \cdot T_{m}\left(u_{m}-\psi\right)^{-} \cdot \operatorname{sg}_{\frac{1}{m}}\left(u_{m}\right) \cdot \exp \left(G\left(u_{m}\right)\right) \cdot T_{k}\left(u_{m}-v_{0}\right)^{+} d x \\
& \quad \leq \int_{\Omega}\left[h(x)+f^{m}(x)+\phi(x) \cdot \frac{l\left(u_{m}\right)}{\bar{a}}\right] \cdot \exp \left(G\left(u_{m}\right)\right) \cdot T_{k}\left(u_{m}-v_{0}\right)^{+} d x
\end{aligned}
$$

where $c$ is a constant such that $0<c<1$, and since $h, f^{m}, \phi \in L^{1}(\Omega)$ we deduce that

$$
\begin{aligned}
& \sum_{i=1}^{N} \int_{\left\{\left|u_{m}-v_{0}\right| \leq k\right\}} a_{i}^{m}\left(x, u_{m}, \nabla u_{m}\right) \cdot \nabla u_{m} \cdot \exp \left(G\left(u_{m}\right) d x\right. \\
& \quad+\int_{\Omega} m \cdot T_{m}\left(u_{m}-\psi\right)^{-} \cdot \operatorname{sg}_{\frac{1}{m}}\left(u_{m}\right) \cdot \exp \left(G\left(u_{m}\right)\right) \cdot T_{k}\left(u_{m}-v_{0}\right)^{+} d x \\
& \quad \leq-c \sum_{i=1}^{N} \int_{\left\{\left|u_{m}-v_{0}\right| \leq k\right\}}\left[a_{i}^{m}\left(x, u_{m}, \nabla u_{m}\right)-a_{i}^{m}\left(x, u_{m}, \frac{\nabla v_{0}}{c}\right)\right] \cdot \nabla\left(u_{m}-\frac{\nabla v_{0}}{c}\right) \cdot \exp \left(G\left(u_{m}\right)\right) d x \\
& \quad+c \sum_{i=1}^{N} \int_{\left\{\left|u_{m}-v_{0}\right| \leq k\right\}} a_{i}^{m}\left(x, u_{m}, \nabla u_{m}\right) \cdot \nabla u_{m} \cdot \exp \left(G\left(u_{m}\right)\right) d x \\
& \quad+c \sum_{i=1}^{N} \int_{\left\{\left|u_{m}-v_{0}\right| \leq k\right\}}\left|a_{i}^{m}\left(x, u_{m}, \frac{\nabla v_{0}}{c}\right)\right| \cdot\left|\nabla\left(u_{m}-\frac{\nabla v_{0}}{c}\right)\right| \cdot \exp \left(G\left(u_{m}\right)\right) d x+c_{1},
\end{aligned}
$$


by (1)

$$
\begin{aligned}
(1-c) & \sum_{i=1}^{N} \int_{\left\{\left|u_{m}-v_{0}\right| \leq k\right\}} a_{i}^{m}\left(x, u_{m}, \nabla u_{m}\right) \cdot \nabla u_{m} \cdot \exp \left(G\left(u_{m}\right)\right) d x \\
& +\int_{\Omega} m \cdot T_{m}\left(u_{m}-\psi\right)^{-} \cdot \operatorname{sg}_{\frac{1}{m}}\left(u_{m}\right) \cdot \exp \left(G\left(u_{m}\right)\right) \cdot T_{k}\left(u_{m}-v_{0}\right)^{+} d x \\
& \leq c \sum_{i=1}^{N} \int_{\left\{\left|u_{m}-v_{0}\right| \leq k\right\}}\left|a_{i}^{m}\left(x, u_{m}, \frac{\nabla v_{0}}{c}\right)\right| \cdot\left|\nabla u_{m}\right| \cdot \exp \left(G\left(u_{m}\right)\right) d x \\
& +c \sum_{i=1}^{N} \int_{\left\{\left|u_{m}-v_{0}\right| \leq k\right\}}\left|a_{i}^{m}\left(x, u_{m}, \frac{\nabla v_{0}}{c}\right)\right| \cdot\left|\frac{\nabla v_{0}}{c}\right| \cdot \exp \left(G\left(u_{m}\right)\right) d x+c_{1},
\end{aligned}
$$

since $\frac{\nabla v_{0}}{c} \in \stackrel{\circ}{W}_{B}^{1}(\Omega)$, and by (8), (3) and (6) and the fact that $\exp (G( \pm \infty)) \leq \exp \left(\frac{\|l\|_{L^{1}(\mathbb{R})}}{\bar{a}}\right)$ we have

$$
\begin{aligned}
(1-c) & \sum_{i=1}^{N} \int_{\left\{\left|u_{m}-v_{0}\right| \leq k\right\}} a_{i}^{m}\left(x, u_{m}, \nabla u_{m}\right) \cdot \nabla u_{m} d x+\int_{\left\{\left|u_{m}-v_{0}\right| \geq 0\right\}} m \cdot T_{m}\left(u_{m}-\psi\right)^{-} \cdot g_{\frac{1}{m}}\left(u_{m}\right) d x \\
& \leq \frac{\tilde{a}(1-c)}{2} \sum_{i=1}^{N} \int_{\left\{\left|u_{m}-v_{0}\right| \leq k\right\}} B_{i}\left(\nabla u_{m}\right) d x+c_{2}(k) \cdot c_{1}
\end{aligned}
$$

where $c_{2}(k)$ is a positive constant which depends only on $k$.

Finally, by (2) we obtain

$$
\sum_{i=1}^{N} \int_{\left\{\left|u_{m}-v_{0}\right| \leq k\right\}} B_{i}\left(\nabla u_{m}\right) d x \leq c_{3} \cdot k
$$

and

$$
0 \leq \int_{\left\{\left|u_{m}-v_{0}\right| \geq 0\right\}} m \cdot T_{m}\left(u_{m}-\psi\right)^{-} \cdot \operatorname{sg}_{\frac{1}{m}}\left(u_{m}\right) d x \leq c_{1} .
$$

Similarly, taking $v=u_{m}-\eta \cdot \exp \left(G\left(u_{m}\right)\right) \cdot T_{k}\left(u_{m}-v_{0}\right)^{-}$as a test function in $\left(\mathcal{P}_{m}\right)$, we obtain

$$
\begin{aligned}
& \sum_{i=1}^{N} \int_{\Omega} a_{i}^{m}\left(x, u_{m}, \nabla u_{m}\right) \cdot \nabla\left(\exp \left(G\left(u_{m}\right)\right) \cdot T_{k}\left(u_{m}-v_{0}\right)^{-}\right) d x \\
& \quad+\sum_{i=1}^{N} \int_{\Omega} b_{i}^{m}\left(x, u_{m}, \nabla u_{m}\right) \cdot \exp \left(-G\left(u_{m}\right)\right) \cdot T_{k}\left(u_{m}-v_{0}\right)^{-} d x \\
& \quad+\int_{\Omega} m \cdot T_{m}\left(u_{m}-\psi\right)^{-} \cdot \operatorname{sg}_{\frac{1}{m}}\left(u_{m}\right) \cdot \exp \left(-G\left(u_{m}\right)\right) \cdot T_{k}\left(u_{m}-v_{0}\right)^{-} d x \\
& \quad \leq \int_{\Omega} f^{m}(x) \cdot \exp \left(-G\left(u_{m}\right)\right) \cdot T_{k}\left(u_{m}-v_{0}\right)^{-} d x
\end{aligned}
$$

and using same techniques, we obtain also

$$
\sum_{i=1}^{N} \int_{\left\{\left|u_{m}-v_{0}\right| \geq k\right\}} B_{i}\left(\nabla u_{m}\right) d x \leq c_{4} \cdot k
$$

and

$$
0 \leq \int_{\left\{\left|u_{m}-v_{0}\right| \leq 0\right\}} m \cdot T_{m}\left(u_{m}-\psi\right)^{-} \cdot \operatorname{sg}_{\frac{1}{m}}\left(u_{m}\right) d x \leq c_{5} .
$$

Additionally, by (26), (27), (28) and (29) we conclude that

$$
\int_{\Omega} B\left(\nabla T_{k}\left(u_{m}\right)\right) d x \leq c \cdot k
$$


with $c_{3}, c_{4}, c_{5}, c_{6}$ being positive constants.

Step 2. Almost everywhere convergence of $\left\{u_{m}\right\}$ :

Firstly, we prove that meas $\left\{x \in \Omega:\left|u_{m}\right| \geq k\right\} \rightarrow 0$.

According to Lemma 2, we have

$$
\begin{aligned}
\left\|T_{k}\left(u_{m}\right)\right\|_{B^{*}} & \leq c\left\|\nabla T_{k}\left(u_{m}\right)\right\|_{B} \\
& \leq c \cdot \epsilon(k) \int_{\Omega} B\left(\nabla T_{k}\left(u_{m}\right)\right) d x \\
& \leq c \cdot \epsilon(k) \cdot k \text { for } k>1,
\end{aligned}
$$

with $c$ being a positive constant and $\epsilon(k) \rightarrow 0$ when $k \rightarrow \infty$. By (31) we obtain

$$
\begin{aligned}
B^{*}\left(\frac{k}{\left\|T_{k}\left(u_{m}\right)\right\|_{B^{*}}}\right) \text { meas }\left\{x \in \Omega:\left|u_{m}\right| \geq k\right\} & \leq \int_{\Omega} B^{*}\left(\frac{T_{k}\left(u_{m}\right)}{\left\|T_{k}\left(u_{m}\right)\right\|_{B^{*}}}\right) d x \\
& \leq \int_{\Omega} B^{*}\left(\frac{k}{\left\|T_{k}\left(u_{m}\right)\right\|_{B^{*}}}\right) d x
\end{aligned}
$$

Thus, we deduce that

$$
B^{*}\left(\frac{k}{\left\|T_{k}\left(u_{m}\right)\right\|_{B^{*}}}\right) \longrightarrow \infty \text { when } k \rightarrow \infty
$$

Hence

$$
\text { meas }\left\{x \in \Omega:\left|u_{m}\right| \geq k\right\} \rightarrow 0 \text { as } k \rightarrow \infty \text { for all } m \in \mathbb{N} .
$$

Secondly we show that for all $\left\{u_{m}\right\}$ measurable function on $\Omega$ such that

$$
T_{k}\left(u_{m}\right) \in \stackrel{\circ}{W}_{B}^{1}(\Omega) \quad \forall k \geq 1,
$$

we have

$$
\operatorname{meas}\left\{x \in \Omega: B\left(\nabla u_{m}\right) \geq \alpha\right\} \rightarrow 0 \text { as } \alpha \rightarrow \infty \text {. }
$$

In the beginning

$$
\begin{aligned}
\operatorname{meas}\left\{x \in \Omega: B\left(\nabla u_{m}\right) \geq 0\right\}= & \operatorname{meas}\left\{\left\{x \in \Omega:\left|u_{m}\right| \geq k, B\left(\nabla u_{m}\right) \geq \alpha\right\}\right. \\
& \left.\cup\left\{x \in \Omega:\left|u_{m}\right|<k, B\left(\nabla u_{m}\right) \geq \alpha\right\}\right\},
\end{aligned}
$$

if we denote

$$
g(\alpha, k)=\operatorname{meas}\left\{x \in \Omega:\left|u_{m}\right| \geq k, B\left(\nabla u_{m}\right) \geq \alpha\right\},
$$

we have

$$
\operatorname{meas}\left\{x \in \Omega:\left|u_{m}\right|<k, B\left(\nabla u_{m}\right) \geq \alpha\right\}=g(\alpha, 0)-g(\alpha, k),
$$

then

$$
\int_{\left\{x \in \Omega:\left|u_{m}\right|<k\right\}} B\left(\nabla u_{m}\right) d x=\int_{0}^{\infty}(g(\alpha, 0)-g(\alpha, k)) d \alpha \leq c . k,
$$

with $\alpha \rightarrow g(\alpha, k)$ is a decreasing map; then

$$
\begin{aligned}
g(\alpha, 0) & \leq \frac{1}{\alpha} \int_{0}^{\alpha} g(s, 0) d s \\
& \leq \frac{1}{\alpha} \int_{0}^{\alpha}(g(s, 0)-g(s, k)) d s+\frac{1}{\alpha} \int_{0}^{\alpha} g(s, k) d s \\
& \leq \frac{1}{\alpha} \int_{0}^{\alpha}(g(s, 0)-g(s, k)) d s+g(0, k),
\end{aligned}
$$


and according to (34) and (35) we have

$$
g(\alpha, 0) \leq \frac{c . k}{\alpha}+g(0, k)
$$

like [28] we obtain

$$
\lim _{k \rightarrow \infty} g(0, k)=0
$$

Hence

$$
g(\alpha, 0) \rightarrow 0 \text { as } \alpha \rightarrow \infty
$$

We have now to demonstrate that the almost everywhere convergence of $\left\{u_{m}:\right\}$

$$
u_{m} \longrightarrow u \text { almost everywhere in } \Omega \text {. }
$$

Let $g(k)=\sup _{m \in \mathbb{N}}$ meas $\left\{x \in \Omega:\left|u_{m}\right|>k\right\} \rightarrow 0$ as $k \rightarrow \infty$. Since $\Omega$ is unbounded domain in $\mathbb{R}^{N}$, we define

$$
\eta_{R}(x)= \begin{cases}1 & \text { if } x<R \\ R+1-\alpha & \text { if } R \leq x<R+1 \\ 0 & \text { if } x \geq R+1\end{cases}
$$

For $R, k>0$, we have by (10)

$$
\begin{aligned}
\int_{\Omega} B\left(\nabla \eta_{R}(|x|) \cdot T_{k}\left(u_{m}\right)\right) d x \leq & c \int_{\left\{x \in \Omega:\left|u_{m}\right|<k\right\}} B\left(\nabla u_{m}\right) d x \\
& +c \int_{\Omega} B\left(T_{k}\left(u_{m}\right) \cdot \nabla \eta_{R}(|x|) d x\right. \\
\leq & c(k, R),
\end{aligned}
$$

which implies that the sequence $\left\{\eta_{R}(|x|) T_{k}\left(u_{m}\right)\right\}$ is bounded in $\stackrel{\circ}{B}_{B}^{1}(\Omega(R+1))$, and by embedding Theorem, for an $N$-function $P$ with $P \ll B$ we have

$$
\stackrel{\circ}{W}_{B}^{1}(\Omega(R+1)) \hookrightarrow L_{P}(\Omega(R+1))
$$

and since $\eta_{R}=1$ in $\Omega(R)$, we have:

$$
\eta_{R} T_{k}\left(u_{m}\right) \longrightarrow v_{k} \text { in } L_{P}(\Omega(R+1)) \text { as } m \longrightarrow \infty .
$$

For $k \in \mathbb{N}^{*}$,

$$
T_{k}\left(u_{m}\right) \longrightarrow v_{k} \text { in } L_{P}(\Omega(R+1)) \text { as } m \longrightarrow \infty
$$

by a diagonal process, we prove that there is measurable $u: \Omega \longrightarrow \mathbb{R}$ such that $u_{m} \longrightarrow u$ a.e. in $\Omega$.

Lemma 3 ([29]). Let an $N$-function $\bar{B}(t)$ satisfy the $\Delta_{2}$-condition and $u_{m}, m \geq 1$ and $u$ be two functions of $L_{B}(\Omega)$ such that

$$
\begin{gathered}
\left\|u_{m}\right\|_{B} \leq c \quad m=1,2, \cdots . \\
u_{m} \longrightarrow \text { u almost everywhere in } \Omega, m \rightarrow \infty .
\end{gathered}
$$

Then,

$$
u_{m} \rightarrow u \text { weakly in } L_{B}(\Omega) \text { as } m \rightarrow \infty .
$$

Hence,

$$
\text { meas }\left\{x \in \Omega:\left|u_{m}\right| \geq k\right\} \longrightarrow 0 \text { when } k \rightarrow \infty \text { for all } m \in \mathbb{N} \text {. }
$$


Step 3. Weak convergence of the gradient:

Since $\mathfrak{W}_{B}^{1}(\Omega)$ reflexive, there exists a subsequence

$$
T_{k}\left(u_{m}\right) \rightarrow v \text { weakly in } \stackrel{\circ}{B}_{B}^{1}(\Omega), \quad m \rightarrow \infty,
$$

and since

$$
\stackrel{\circ}{W}_{B}^{1}(\Omega) \hookrightarrow L_{B}(\Omega),
$$

we have

$$
\nabla T_{k}\left(u_{m}\right) \rightarrow \nabla u_{m} \text { in } L_{B}(\Omega) \text { as } m \rightarrow \infty,
$$

since

$$
u_{m} \longrightarrow u \text { almost everywhere in } \Omega \text { as } m \rightarrow \infty,
$$

implies the local convergence in measure and, therefore, the local Cauchy property of $u_{m}$ in measure

$$
\operatorname{meas}\left\{\Omega(R):\left|u_{m}-u_{n}\right| \geq k\right\} \rightarrow 0 \text { as } m, n \rightarrow \infty \text { for any } k>0 \text {. }
$$

Proving that

$$
\nabla u_{m} \longrightarrow \nabla u \text { locally in measure as } m \rightarrow \infty \text {. }
$$

For that, we borrow ideas from Evans [13], Demangel-Hebey [12] and Koznikova L. M. [21,22]. Let $\delta>0$ be given. By Egoroff's Theorem, there exists $E_{\delta, k, \alpha} \subset \subset \Omega$ such that

$$
\begin{gathered}
E_{\delta, k, \alpha}(R)=\left\{\Omega(R):\left|u_{m}-u_{n}\right|<k, B\left(\nabla u_{m}\right)<\alpha, B\left(\nabla u_{n}\right)<\alpha,\left|u_{m}\right| \leq \alpha,\right. \\
\left.\left|u_{n}\right| \leq \alpha,\left|\nabla\left(u_{m}-u_{n}\right)\right| \geq \delta\right\} \\
\left\{\Omega(R):\left|\nabla\left(u_{m}-u_{n}\right)\right| \geq \delta\right\} \subset\left\{\Omega: B\left(\nabla u_{m}\right)>\alpha\right\} \cup\left\{\Omega: B\left(\nabla u_{n}\right)>\alpha\right\} \\
\cup\left\{\Omega(R):\left|u_{m}-u_{n}\right| \geq k\right\} \cup\left\{\Omega:\left|u_{m}\right|>\alpha\right\} \\
\cup\left\{\Omega:\left|u_{n}\right|>\alpha\right\} \cup E_{\delta, k, \alpha}(R) .
\end{gathered}
$$

Then, by Lemma 3 and (33) we obtain that

$$
\text { meas } \begin{aligned}
\left.\Omega(R):\left|\nabla\left(u_{m}-u_{n}\right)\right| \geq \delta\right\} \leq & 4 \epsilon+\text { meas } E_{\delta, k, \alpha}(R) \\
& + \text { meas }\left\{\Omega(R):\left|u_{m}-u_{n}\right| \geq k\right\} \forall n, m \in \mathbb{N}^{*} .
\end{aligned}
$$

According to (1) and the fact that a continuous function on a compact set achieves the lowest value, there exists a function $\theta(x)>0$ almost everywhere in $\Omega$, such that, for $B(\xi) \leq \alpha, B\left(\xi^{\prime}\right) \leq \alpha,|s| \leq \alpha$ and for $i=1, \cdots, N,\left|\xi_{i}-\xi_{i}^{\prime}\right| \geq k$, we have that

$$
\sum_{i=1}^{N}\left[a_{i}^{m}(x, s, \xi)-a_{i}^{m}\left(x, s, \xi^{\prime}\right)\right] \cdot\left(\xi_{i}-\xi_{i}^{\prime}\right) \geq \theta(x),
$$

holds. Writing $\left(\mathcal{P}_{m}\right)$ twice for $\left\{u_{m}\right\}$ and $\left\{u_{n}\right\}$, and by subtracting the second relation from the first and according to (23), (27), (29) and (36) we obtain

$$
\sum_{i=1}^{N} \int_{\Omega}\left[a_{i}^{m}\left(x, u_{m}, \nabla u_{m}\right)-a_{i}^{m}\left(x, u_{n}, \nabla u_{n}\right)\right] \cdot \nabla\left(u_{m}-u_{n}-v\right) d x=0
$$

Consider the following test function:

$$
v=u_{m}-u_{n}-\eta_{R}(|x|) \eta_{\alpha}\left(\left|u_{n}\right|\right) \eta_{\alpha}\left(\left|u_{m}\right|\right) \exp \left(G\left(\left|u_{m}-u_{n}\right|\right)\right) T_{\delta}\left(u_{m}-u_{n}\right)
$$


Further on, by applying (40), we get

with

$$
\begin{aligned}
\int_{E_{\delta, k, \alpha}(R)} \theta(x) d x \leq & \sum_{i=1}^{N} \int_{E_{\delta, k, \alpha}(R)}\left[a_{i}^{m}\left(x, u_{m}, \nabla u_{m}\right)-a_{i}^{m}\left(x, u_{m}, \nabla u_{n}\right)\right] \\
& \times \nabla\left(\eta_{R}(|x|) \eta_{\alpha}\left(\left|u_{n}\right|\right) \eta_{\alpha}\left(\left|u_{m}\right|\right) \exp \left(G\left(\left|u_{m}-u_{n}\right|\right)\right) T_{\delta}\left(u_{m}-u_{n}\right)\right) d x \\
& \leq \sum_{i=1}^{N} \int_{\left\{\Omega:\left|u_{m}-u_{n}\right|<k\right\}} \eta_{R}(|x|) \eta_{\alpha}\left(\left|u_{n}\right|\right) \eta_{\alpha}\left(\left|u_{m}\right|\right) \exp \left(G\left(\left|u_{m}-u_{n}\right|\right)\right) \\
& \times\left[a_{i}^{m}\left(x, u_{m}, \nabla u_{m}\right)-a_{i}^{m}\left(x, u_{m}, \nabla u_{n}\right)\right] \cdot \nabla\left(u_{m}-u_{n}\right) d x \\
= & A_{1}^{m}(x)+A_{2}^{m}(x),
\end{aligned}
$$

$$
\begin{aligned}
A_{1}^{m}(x)= & \sum_{i=1}^{N} \int_{\left\{\left|u_{m}-u_{n}\right|<k\right\}} \eta_{R}(|x|) \eta_{\alpha}\left(\left|u_{n}\right|\right) \eta_{\alpha}\left(\left|u_{m}\right|\right) \exp \left(G\left(\left|u_{m}-u_{n}\right|\right)\right) \\
& \times\left[a_{i}^{m}\left(x, u_{m}, \nabla u_{m}\right)-a_{i}^{m}\left(x, u_{n}, \nabla u_{n}\right)\right] \cdot \nabla\left(u_{m}-u_{n}\right) d x
\end{aligned}
$$

and

$$
\begin{aligned}
A_{2}^{m}(x)= & \sum_{i=1}^{N} \int_{\left\{\left|u_{m}-u_{n}\right|<k\right\}} \eta_{R}(|x|) \eta_{\alpha}\left(\left|u_{n}\right|\right) \eta_{\alpha}\left(\left|u_{m}\right|\right) \exp \left(G\left(\left|u_{m}-u_{n}\right|\right)\right) \\
& \times\left[a_{i}^{m}\left(x, u_{n}, \nabla u_{n}\right)-a_{i}^{m}\left(x, u_{m}, \nabla u_{n}\right)\right] \cdot \nabla\left(u_{m}-u_{n}\right) d x
\end{aligned}
$$

Since $B(u)$ satisfies the $\Delta_{2}$-condition, by (14) we have

$$
\int_{\Omega} B(u) d x \leq c_{0}\|u\|_{B, \Omega}
$$

According to Lemma 3, we get

$$
\left\|u_{m}\right\|_{\stackrel{\complement}{B}_{B}^{1}(\Omega)} \leq c_{1} \quad m \in \mathbb{N}^{*},
$$

and

$$
\left\|B\left(\nabla u_{m}\right)\right\|_{1} \leq c_{2} \quad m \in \mathbb{N}^{*} .
$$

Additionally, using (14) and (3) we have

$$
\begin{aligned}
\|a(x, u, \nabla u)\|_{L_{\bar{B}}(\Omega)} & =\sum_{i=1}^{N}\left\|a_{i}(x, u, \nabla u)\right\|_{L_{\bar{B}_{i}}(\Omega)} \\
& \leq \sum_{i=1}^{N} \int_{\Omega} \bar{B}_{i}\left(a_{i}(x, u, \nabla u)\right) d x+N \\
& \leq c_{3}\|B(u)\|_{1, \Omega}+\|\varphi\|_{1, \Omega}+N \\
& \leq c_{4} .
\end{aligned}
$$


Hence,

$$
\begin{aligned}
A_{1}^{m}(x)= & \sum_{i=1}^{N} \int_{\left\{\Omega:\left|u_{m}-u_{n}\right|<k,|x|<R,\left|u_{m}\right|<\alpha,\left|u_{n}\right|<\alpha\right\}} \exp \left(G\left(\left|u_{m}-u_{n}\right|\right)\right) \\
& \times\left[a_{i}^{m}\left(x, u_{m}, \nabla u_{m}\right)-a_{i}^{m}\left(x, u_{n}, \nabla u_{n}\right)\right] \cdot \nabla\left(u_{m}-u_{n}\right) d x \\
& +\sum_{i=1}^{N} \int_{\left\{\Omega:\left|u_{m}-u_{n}\right|<k, R \leq|x| \leq R+1, \alpha \leq\left|u_{m}\right| \leq \alpha+1, \alpha \leq\left|u_{n}\right| \leq \alpha+1\right\}}(R+1-|x|) \\
& \times\left(\alpha+1-\left|u_{n}\right|\right) \cdot\left(\alpha+1-\left|u_{m}\right|\right) \cdot \exp \left(G\left(\left|u_{m}-u_{n}\right|\right)\right) \\
& \times\left[a_{i}^{m}\left(x, u_{m}, \nabla u_{m}\right)-a_{i}^{m}\left(x, u_{n}, \nabla u_{n}\right)\right] \cdot \nabla\left(u_{m}-u_{n}\right) d x,
\end{aligned}
$$

since $\exp (G( \pm \infty)) \leq \exp \left(\frac{\|l\|_{L^{1}(\mathbb{R}}}{\bar{a}}\right)$, and according to (42), (43), (44) and (15) we obtain that

$$
A_{1}^{m}(x) \leq c_{1}(R, \alpha) \cdot k
$$

the same for $A_{2}^{m}(x)$. We get

$$
A_{2}^{m}(x) \leq c_{2}(R, \alpha) \cdot k
$$

Then,

$$
\int_{E_{\delta, k, \alpha}(R)} \theta(x) d x \leq c_{3}(R, \alpha) \cdot k
$$

For any arbitrary $\delta>0$ for fixed $m$ and $\alpha$, by choosing $k$ from (45) we establish the following inequality

$$
\int_{E_{\delta, k, \alpha}(R)} \theta(x) d x<\delta
$$

By applying Lemma 1, for any $\epsilon>0$, we find

$$
\text { meas } E_{\delta, k, \alpha}(R)<\epsilon .
$$

In addition, according to (37), we have

$$
\text { meas }\left\{\Omega(R):\left|u_{m}-u_{n}\right| \geq k\right\}<\epsilon, m, n>0 .
$$

By combining (39), (46) and (47) we deduce the inequality

$$
\text { meas }\left\{\Omega(R):\left|\nabla\left(u_{m}-u_{n}\right)\right| \geq \delta\right\}<6 \epsilon, n, m>0 .
$$

Hence, the sequence $\left\{\nabla u_{m}\right\}$ is fundamental in measure on the set $\Omega(R)$ for any $R>0$. This implies (38) and the selective convergence,

$$
\nabla u_{m} \longrightarrow \nabla u \text { almost everywhere in } \Omega, m \rightarrow \infty .
$$

Then, we obtain for any fixed $k>0$

$$
\nabla T_{k}\left(u_{m}\right) \longrightarrow \nabla T_{k}(u) \text { almost everywhere in } \Omega \text { as } m \rightarrow \infty .
$$

Applying Lemma 3, we have the following weak convergence

$$
\nabla T_{k}\left(u_{m}\right) \rightarrow \nabla T_{k}(u) \text { in } L_{B}(\Omega) \text { as } m \rightarrow \infty .
$$

Proposition 4. Suppose that Conditions (1)-(4) are satisfied and let $\left(u_{m}\right)_{m \in \mathbb{N}}$ be a sequence in $\mathrm{W}_{B}^{1}(\Omega(R))$ such that 
(a) $u_{m} \rightarrow u$ in $\stackrel{\circ}{B}_{B}^{1}(\Omega(R))$

(b) $a^{m}\left(x, u_{m}, \nabla u_{m}\right)$ is bounded in $L_{\bar{B}}(\Omega(R))$

(c) $\sum_{i=1}^{N} \int_{\Omega(R)}\left[a_{i}^{m}\left(x, u_{m}, \nabla u_{m}\right)-a_{i}^{m}\left(x, u_{m}, \nabla u \chi_{\epsilon}\right) . \nabla\left(u_{m}-u \chi_{\epsilon}\right) d x \rightarrow 0\right.$ as $\epsilon \rightarrow+\infty$ ( $\chi_{\epsilon}$ the characteristic function of $\left.\Omega_{\epsilon}(R)=\{x \in \Omega ;|\nabla u| \leq \epsilon\}\right)$. Then

$$
B\left(\left|\nabla u_{m}\right|\right) \longrightarrow B(|\nabla u|) \text { in } L^{1}(\Omega(R))
$$

Proof. Let $\epsilon>0$ fixed, and $\eta>\epsilon$; then from (1) we have

$$
\begin{aligned}
0 & \leq \sum_{i=1}^{N} \int_{\Omega_{\eta}(R)}\left[a_{i}^{m}\left(x, u_{m}, \nabla u_{m}\right)-a_{i}^{m}\left(x, u_{m}, \nabla u\right)\right] \cdot \nabla\left(u_{m}-u\right) d x \\
& \leq \sum_{i=1}^{N} \int_{\Omega_{\epsilon}(R)}\left[a_{i}^{m}\left(x, u_{m}, \nabla u_{m}\right)-a_{i}^{m}\left(x, u_{m}, \nabla u\right)\right] \cdot \nabla\left(u_{m}-u\right) d x \\
& =\sum_{i=1}^{N} \int_{\Omega_{\epsilon}(R)}\left[a_{i}^{m}\left(x, u_{m}, \nabla u_{m}\right)-a_{i}^{m}\left(x, u_{m}, \nabla u \chi_{\epsilon}\right)\right] \cdot \nabla\left(u_{m}-u \chi_{\epsilon}\right) d x \\
& \leq \sum_{i=1}^{N} \int_{\Omega(R)}\left[a_{i}^{m}\left(x, u_{m}, \nabla u_{m}\right)-a_{i}^{m}\left(x, u_{m}, \nabla u \chi_{\epsilon}\right)\right] \cdot \nabla\left(u_{m}-u \chi_{\epsilon}\right) d x,
\end{aligned}
$$

using the condition $(c)$ we get

$$
\lim _{m \rightarrow \infty} \sum_{i=1}^{N} \int_{\Omega(R)}\left[a_{i}^{m}\left(x, u_{m}, \nabla u_{m}\right)-a_{i}^{m}\left(x, u_{m}, \nabla u \chi_{\epsilon}\right)\right] \cdot \nabla\left(u_{m}-u \chi_{\epsilon}\right) d x=0
$$

proceeding as in [28], and we obtain $\nabla u_{m} \longrightarrow \nabla u$; by letting $\epsilon \longrightarrow \infty$ we get

$$
\nabla u_{m} \chi_{\epsilon} \longrightarrow \nabla u
$$

Thus, since

$$
\begin{aligned}
& \sum_{i=1}^{N} \int_{\Omega(R)} a_{i}^{m}\left(x, u_{m}, \nabla u_{m}\right) \cdot \nabla u_{m} d x \\
& \quad=\sum_{i=1}^{N} \int_{\Omega(R)}\left[a_{i}^{m}\left(x, u_{m}, \nabla u_{m}\right)-a_{i}^{m}\left(x, u_{m}, \nabla u \chi_{\epsilon}\right)\right] \cdot \nabla\left(u_{m}-u \chi_{\epsilon}\right) d x \\
& \quad+\sum_{i=1}^{N} \int_{\Omega(R)} a_{i}^{m}\left(x, u_{m}, \nabla \chi_{\epsilon}\right) \cdot \nabla\left(u_{m}-u \chi_{\epsilon}\right) d x+\sum_{i=1}^{N} \int_{\Omega(R)} a_{i}^{m}\left(x, u_{m}, \nabla u_{m}\right) \cdot \nabla u \chi_{\epsilon} d x,
\end{aligned}
$$

using $(b)$, we have

$$
\sum_{i=1}^{N} a_{i}^{m}\left(x, u_{m}, \nabla u_{m}\right) \rightarrow \sum_{i=1}^{N} a_{i}^{m}(x, u, \nabla u) \text { weakly in }\left(L_{\bar{B}}(\Omega(R))\right)^{N}
$$

Therefore

$$
\sum_{i=1}^{N} \int_{\Omega(R)} a_{i}^{m}\left(x, u_{m}, \nabla u_{m}\right) . \nabla u \chi_{\epsilon} d x \longrightarrow \sum_{i=1}^{N} \int_{\Omega(R)} a_{i}^{m}(x, u, \nabla u) . \nabla u d x \text { as } m \rightarrow \infty, \epsilon \rightarrow \infty .
$$


Thus,

$$
\sum_{i=1}^{N} \int_{\Omega(R)}\left[a_{i}^{m}\left(x, u_{m}, \nabla u_{m}\right)-a_{i}^{m}\left(x, u_{m}, \nabla u \chi_{\epsilon}\right)\right] . \nabla\left(u_{m}-\nabla u \chi_{\epsilon}\right) d x \longrightarrow 0 \text { as } m \rightarrow \infty, \epsilon \rightarrow \infty .
$$

and

$$
\sum_{i=1}^{N} \int_{\Omega(R)} a_{i}^{m}\left(x, u_{m}, \nabla u \chi_{\epsilon}\right) \cdot \nabla\left(u_{m}-u \chi_{\epsilon}\right) d x \longrightarrow 0 \text { as } m \rightarrow \infty, \epsilon \rightarrow \infty
$$

Thus,

$$
\lim _{m \rightarrow \infty} \sum_{i=1}^{N} \int_{\Omega(R)} a_{i}^{m}\left(x, u_{m}, \nabla u_{m}\right) . \nabla u_{m} d x=\sum_{i=1}^{N} \int_{\Omega(R)} a_{i}^{m}(x, u, \nabla u) . \nabla u d x,
$$

from (2), and the vitali's Theorem, we get

$$
\bar{a} \sum_{i=1}^{N} \int_{\Omega(R)} B_{i}\left(\left|\nabla u_{m}\right|\right) d x-\int_{\Omega(R)} \phi(x) d x \geq \bar{a} \sum_{i=1}^{N} \int_{\Omega(R)} B_{i}(|\nabla u|) d x-\int_{\Omega(R)} \phi(x) d x,
$$

Consequently, by Lemma 2.6 in [11] and (48), we get

$$
B\left(\left|\nabla u_{m}\right|\right) \longrightarrow B(|\nabla u|) \text { in } \stackrel{\circ}{W}_{B}^{1}(\Omega(R)),
$$

thanks to lemma 1 (see [20]) and (48), we have

$$
B\left(\left|\nabla u_{m}\right|\right) \longrightarrow B(|\nabla u|) \text { in } L^{1}(\Omega(R)) .
$$

Step 4. Strong convergence of the gradient:

In this step we consider again the following test function:

$$
v=u_{m}+\eta \exp \left(G\left(\left|u_{m}\right|\right)\right)\left(T_{k}\left(u_{m}\right)-T_{k}(u)\right) \eta_{j}\left(\left|u_{m}\right|\right) h_{j}\left(u_{m}\right),
$$

with, $\left.h_{j}\left(u_{m}\right)\right)=1-\left|T_{1}\left(u_{m}-T_{j}\left(u_{m}\right)\right)\right|= \begin{cases}1 & \text { if }\left\{\left|u_{m}\right| \geq j\right\} \\ 0 & \text { if }\left\{\left|u_{m}\right| \geq j+1\right\} \\ j+1-\left|u_{m}\right| & \text { if }\left\{j \leq\left|u_{m}\right| \leq j+1\right\}\end{cases}$

and, $\left|T_{k}\left(u_{m}\right)-T_{k}(u)\right|$ at the same sign when $u_{m} \in\left\{\left|u_{m}\right|>k\right\}$, where $j \geq k>0$ and $\eta$ is small enough, we obtain

$$
\begin{aligned}
& \sum_{i=1}^{N} \int_{\Omega} a_{i}^{m}\left(x, u_{m}, \nabla u_{m}\right) \cdot \nabla\left(\exp \left(G\left(\left|u_{m}\right|\right)\right)\left(T_{k}\left(u_{m}\right)-T_{k}(u)\right) \eta_{j}\left(\left|u_{m}\right|\right) h_{j}\left(u_{m}\right)\right) d x \\
& \quad+\int_{\Omega} m \cdot T_{m}\left(u_{m}-\psi\right)^{-} \cdot \operatorname{sg}_{\frac{1}{m}}\left(u_{m}\right) \cdot \exp \left(G\left(\left|u_{m}\right|\right)\right)\left(T_{k}\left(u_{m}\right)-T_{k}(u)\right) \eta_{j}\left(\left|u_{m}\right|\right) h_{j}\left(u_{m}\right) d x \\
& \quad+\sum_{i=1}^{N} \int_{\Omega} b_{i}^{m}\left(x, u_{m}, \nabla u_{m}\right) \cdot \exp \left(G\left(\left|u_{m}\right|\right)\right)\left(T_{k}\left(u_{m}\right)-T_{k}(u)\right) \eta_{j}\left(\left|u_{m}\right|\right) h_{j}\left(u_{m}\right) d x \\
& \quad \leq \int_{\Omega} f^{m}(x) \cdot \exp \left(G\left(\left|u_{m}\right|\right)\right)\left(T_{k}\left(u_{m}\right)-T_{k}(u)\right) \eta_{j}\left(\left|u_{m}\right|\right) h_{j}\left(u_{m}\right) d x
\end{aligned}
$$


which implies,

$$
\begin{aligned}
& \sum_{i=1}^{N} \int_{\Omega} a_{i}^{m}\left(x, u_{m}, \nabla u_{m}\right) \cdot \nabla\left(\exp \left(G\left(\left|u_{m}\right|\right)\right)\right)\left(T_{k}\left(u_{m}\right)-T_{k}(u)\right) \eta_{j}\left(\left|u_{m}\right|\right) h_{j}\left(u_{m}\right) d x \\
& \quad+\sum_{i=1}^{N} \int_{\Omega} a_{i}^{m}\left(x, u_{m}, \nabla u_{m}\right) \cdot \exp \left(G\left(\left|u_{m}\right|\right)\right) \nabla\left(\left(T_{k}\left(u_{m}\right)-T_{k}(u)\right)\right) \eta_{j}\left(\left|u_{m}\right|\right) h_{j}\left(u_{m}\right) d x \\
& \quad+\sum_{i=1}^{N} \int_{\Omega} a_{i}^{m}\left(x, u_{m}, \nabla u_{m}\right) \cdot \exp \left(G\left(\left|u_{m}\right|\right)\right)\left(T_{k}\left(u_{m}\right)-T_{k}(u)\right) \nabla \eta_{j}\left(\left|u_{m}\right|\right) h_{j}\left(u_{m}\right) d x \\
& \left.\quad+\sum_{i=1}^{N} \int_{\Omega} a_{i}^{m}\left(x, u_{m}, \nabla u_{m}\right) \cdot \exp \left(G\left(\left|u_{m}\right|\right)\right)\right)\left(T_{k}\left(u_{m}\right)-T_{k}(u)\right) \eta_{j}\left(\left|u_{m}\right|\right) \nabla h_{j}\left(u_{m}\right) d x \\
& \quad+\sum_{i=1}^{N} \int_{\Omega} b_{i}^{m}\left(x, u_{m}, \nabla u_{m}\right) \cdot \exp \left(G\left(\left|u_{m}\right|\right)\right)\left(T_{k}\left(u_{m}\right)-T_{k}(u)\right) \eta_{j}\left(\left|u_{m}\right|\right) h_{j}\left(u_{m}\right) d x \\
& \quad+\int_{\Omega} m \cdot T_{m}\left(u_{m}-\psi\right)^{-} \cdot g_{\frac{1}{m}}\left(u_{m}\right) \cdot \exp \left(G\left(\left|u_{m}\right|\right)\right)\left(T_{k}\left(u_{m}\right)-T_{k}(u)\right) \eta_{j}\left(\left|u_{m}\right|\right) h_{j}\left(u_{m}\right) d x \\
& \quad \leq \int_{\Omega} f^{m}(x) \cdot \exp \left(G\left(\left|u_{m}\right|\right)\right)\left(T_{k}\left(u_{m}\right)-T_{k}(u)\right) \eta_{j}\left(\left|u_{m}\right|\right) h_{j}\left(u_{m}\right) d x
\end{aligned}
$$

then,

$$
\begin{aligned}
& \sum_{i=1}^{N} \int_{\Omega} a_{i}^{m}\left(x, u_{m}, \nabla u_{m}\right) \cdot \nabla u_{m} \cdot \frac{l\left(\left|u_{m}\right|\right)}{\bar{a}} \cdot \exp \left(G\left(\left|u_{m}\right|\right)\right)\left(T_{k}\left(u_{m}\right)-T_{k}(u)\right) \eta_{j}\left(\left|u_{m}\right|\right) h_{j}\left(u_{m}\right) d x \\
& \quad+\sum_{i=1}^{N} \int_{\Omega} a_{i}^{m}\left(x, u_{m}, \nabla u_{m}\right) \cdot \exp \left(G\left(\left|u_{m}\right|\right)\right) \nabla\left(\left(T_{k}\left(u_{m}\right)-T_{k}(u)\right)\right) \eta_{j}\left(\left|u_{m}\right|\right) h_{j}\left(u_{m}\right) d x \\
& \quad+\sum_{i=1}^{N} \int_{\Omega} a_{i}^{m}\left(x, u_{m}, \nabla u_{m}\right) \cdot \exp \left(G\left(\left|u_{m}\right|\right)\right)\left(T_{k}\left(u_{m}\right)-T_{k}(u)\right) \nabla \eta_{j}\left(\left|u_{m}\right|\right) h_{j}\left(u_{m}\right) d x \\
& \left.\quad+\sum_{i=1}^{N} \int_{\Omega} a_{i}^{m}\left(x, u_{m}, \nabla u_{m}\right) \cdot \exp \left(G\left(\left|u_{m}\right|\right)\right)\right)\left(T_{k}\left(u_{m}\right)-T_{k}(u)\right) \eta_{j}\left(\left|u_{m}\right|\right) \nabla h_{j}\left(u_{m}\right) d x \\
& \quad+\int_{\Omega} m \cdot T_{m}\left(u_{m}-\psi\right)^{-} \cdot \operatorname{sg}_{\frac{1}{m}}\left(u_{m}\right) \cdot \exp \left(G\left(\left|u_{m}\right|\right)\right)\left(T_{k}\left(u_{m}\right)-T_{k}(u)\right) \eta_{j}\left(\left|u_{m}\right|\right) h_{j}\left(u_{m}\right) d x \\
& \quad \leq \sum_{i=1}^{N} \int_{\Omega}\left|b_{i}^{m}\left(x, u_{m}, \nabla u_{m}\right)\right| \cdot \exp \left(G\left(\left|u_{m}\right|\right)\right)\left(T_{k}\left(u_{m}\right)-T_{k}(u)\right) \eta_{j}\left(\left|u_{m}\right|\right) h_{j}\left(u_{m}\right) d x \\
& \quad+\int_{\Omega} f^{m}(x) \cdot \exp \left(G\left(\left|u_{m}\right|\right)\right)\left(T_{k}\left(u_{m}\right)-T_{k}(u)\right) \eta_{j}\left(\left|u_{m}\right|\right) h_{j}\left(u_{m}\right) d x,
\end{aligned}
$$


by (2) and (4) we get

$$
\begin{aligned}
\bar{a} \sum_{i=1}^{N} & \int_{\Omega} B_{i}\left(\left|\nabla u_{m}\right|\right) \cdot \frac{l\left(\left|u_{m}\right|\right)}{\bar{a}} \cdot \exp \left(G\left(\left|u_{m}\right|\right)\right)\left(T_{k}\left(u_{m}\right)-T_{k}(u)\right) \eta_{j}\left(\left|u_{m}\right|\right) h_{j}\left(u_{m}\right) d x \\
& +\sum_{i=1}^{N} \int_{\Omega} a_{i}^{m}\left(x, u_{m}, \nabla u_{m}\right) \cdot \exp \left(G\left(\left|u_{m}\right|\right)\right) \nabla\left(\left(T_{k}\left(u_{m}\right)-T_{k}(u)\right)\right) \eta_{j}\left(\left|u_{m}\right|\right) h_{j}\left(u_{m}\right) d x \\
& +\sum_{i=1}^{N} \int_{\Omega} a_{i}^{m}\left(x, u_{m}, \nabla u_{m}\right) \cdot \exp \left(G\left(\left|u_{m}\right|\right)\right)\left(T_{k}\left(u_{m}\right)-T_{k}(u)\right) \nabla \eta_{j}\left(\left|u_{m}\right|\right) h_{j}\left(u_{m}\right) d x \\
& \left.+\sum_{i=1}^{N} \int_{\Omega} a_{i}^{m}\left(x, u_{m}, \nabla u_{m}\right) \cdot \exp \left(G\left(\left|u_{m}\right|\right)\right)\right)\left(T_{k}\left(u_{m}\right)-T_{k}(u)\right) \eta_{j}\left(\left|u_{m}\right|\right) \nabla h_{j}\left(u_{m}\right) d x \\
& +\int_{\Omega} m \cdot T_{m}\left(u_{m}-\psi\right)^{-} \cdot g_{\frac{1}{m}}\left(u_{m}\right) \cdot \exp \left(G\left(\left|u_{m}\right|\right)\right)\left(T_{k}\left(u_{m}\right)-T_{k}(u)\right) \eta_{j}\left(\left|u_{m}\right|\right) h_{j}\left(u_{m}\right) d x \\
& \leq \sum_{i=1}^{N} \int_{\Omega} B_{i}\left(\left|\nabla u_{m}\right|\right) l\left(\left|u_{m}\right|\right) \cdot \exp \left(G\left(\left|u_{m}\right|\right)\right)\left(T_{k}\left(u_{m}\right)-T_{k}(u)\right) \eta_{j}\left(\left|u_{m}\right|\right) h_{j}\left(u_{m}\right) d x \\
& +\int_{\Omega}\left(h(x)+f^{m}(x)+\phi(x) \cdot \frac{l\left(\left|u_{m}\right|\right)}{\bar{a}}\right) \cdot \exp \left(G\left(\left|u_{m}\right|\right)\right)\left(T_{k}\left(u_{m}\right)-T_{k}(u)\right) \eta_{j}\left(\left|u_{m}\right|\right) h_{j}\left(u_{m}\right) d x
\end{aligned}
$$

we then obtain

$$
\begin{aligned}
& \sum_{i=1}^{N} \int_{\left\{\left|u_{m}\right| \leq j\right\}} a_{i}^{m}\left(x, u_{m}, \nabla u_{m}\right) \cdot \exp \left(G\left(\left|u_{m}\right|\right)\right)\left(\nabla T_{k}\left(u_{m}\right)-\nabla T_{k}(u)\right) \eta_{j}\left(\left|u_{m}\right|\right) d x \\
& \quad-\sum_{i=1}^{N} \int_{\left\{j \leq\left|u_{m}\right| \leq j+1\right\}} a_{i}^{m}\left(x, u_{m}, \nabla u_{m}\right) \cdot \nabla u_{m} \cdot\left(j+1+\left|u_{m}\right|\right) \cdot \nabla T_{k}(u) \eta_{j}\left(\left|u_{m}\right|\right) \exp \left(G\left(\left|u_{m}\right|\right)\right) d x \\
& \quad+\sum_{i=1}^{N} \int_{\Omega} a_{i}^{m}\left(x, u_{m}, \nabla u_{m}\right) \cdot \exp \left(G\left(\left|u_{m}\right|\right)\right)\left(T_{k}\left(u_{m}\right)-T_{k}(u)\right) \nabla \eta_{j}\left(\left|u_{m}\right|\right) h_{j}\left(u_{m}\right) d x \\
& \quad+\sum_{i=1}^{N} \int_{\left\{j \leq\left|u_{m}\right| \leq j+1\right\}} a_{i}^{m}\left(x, u_{m}, \nabla u_{m}\right) \cdot \nabla u_{m} \cdot \exp \left(G\left(\left|u_{m}\right|\right)\right)\left(T_{k}\left(u_{m}\right)-T_{k}(u)\right) \eta_{j}\left(\left|u_{m}\right|\right) d x \\
& \quad+\int_{\Omega} m \cdot T_{m}\left(u_{m}-\psi\right)^{-} \cdot s_{\frac{1}{m}}\left(u_{m}\right) \cdot \exp \left(G\left(\left|u_{m}\right|\right)\right)\left(T_{k}\left(u_{m}\right)-T_{k}(u)\right) \eta_{j}\left(\left|u_{m}\right|\right) h_{j}\left(u_{m}\right) d x \\
& \quad \leq \int_{\Omega}\left(h(x)+f^{m}(x)+\phi(x) \cdot \frac{l\left(\left|u_{m}\right|\right)}{\bar{a}}\right) \cdot \exp \left(G\left(\left|u_{m}\right|\right)\right)\left(T_{k}\left(u_{m}\right)-T_{k}(u)\right) \eta_{j}\left(\left|u_{m}\right|\right) h_{j}\left(u_{m}\right) d x
\end{aligned}
$$


By (2) we get

$$
\begin{aligned}
& \sum_{i=1}^{N} \int_{\left\{\left|u_{m}\right| \leq j\right\}} a_{i}^{m}\left(x, u_{m}, \nabla u_{m}\right) \cdot \exp \left(G\left(\left|u_{m}\right|\right)\right)\left(\nabla T_{k}\left(u_{m}\right)-\nabla T_{k}(u)\right) \eta_{j}\left(\left|u_{m}\right|\right) d x \\
& \quad+\sum_{i=1}^{N} \int_{\Omega} a_{i}^{m}\left(x, u_{m}, \nabla u_{m}\right) \cdot \exp \left(G\left(\left|u_{m}\right|\right)\right)\left(T_{k}\left(u_{m}\right)-T_{k}(u)\right) \nabla \eta_{j}\left(\left|u_{m}\right|\right) h_{j}\left(u_{m}\right) d x \\
& \quad+\int_{\Omega} m \cdot T_{m}\left(u_{m}-\psi\right)^{-} \cdot g_{\frac{1}{m}}\left(u_{m}\right) \cdot \exp \left(G\left(\left|u_{m}\right|\right)\right)\left(T_{k}\left(u_{m}\right)-T_{k}(u)\right) \eta_{j}\left(\left|u_{m}\right|\right) h_{j}\left(u_{m}\right) d x \\
& \quad \leq \int_{\Omega}\left(h(x)+f^{m}(x)+\phi(x) \cdot \frac{l\left(\left|u_{m}\right|\right)}{\bar{a}}\right) \cdot \exp \left(G\left(\left|u_{m}\right|\right)\right)\left(T_{k}\left(u_{m}\right)-T_{k}(u)\right) \eta_{j}\left(\left|u_{m}\right|\right) h_{j}\left(u_{m}\right) d x \\
& \quad-\sum_{i=1}^{N} \int_{\left\{j \leq\left|u_{m}\right| \leq j+1\right\}} \phi(x) \cdot\left(j+1+\left|u_{m}\right|\right) \cdot \nabla T_{k}(u) \exp \left(G\left(\left|u_{m}\right|\right)\right) \eta_{j}\left(\left|u_{m}\right|\right) d x \\
& \quad+\sum_{i=1}^{N} \int_{\left\{j \leq\left|u_{m}\right| \leq j+1\right\}} \phi(x) \cdot \exp \left(G\left(\left|u_{m}\right|\right)\right)\left(T_{k}\left(u_{m}\right)-T_{k}(u)\right) \eta_{j}\left(\left|u_{m}\right|\right) d x \\
& \quad+\bar{a} \sum_{i=1}^{N} \int_{\left\{j \leq\left|u_{m}\right| \leq j+1\right\}} B_{i}\left(\left|\nabla u_{m}\right|\right)\left(j+1+\left|u_{m}\right|\right) \cdot \nabla T_{k}(u) \exp \left(G\left(\left|u_{m}\right|\right)\right) \eta_{j}\left(\left|u_{m}\right|\right) d x \\
& \quad-\bar{a} \sum_{i=1}^{N} \int_{\left\{j \leq\left|u_{m}\right| \leq j+1\right\}} B_{i}\left(\left|\nabla u_{m}\right|\right) \exp \left(G\left(\left|u_{m}\right|\right)\right)\left(T_{k}\left(u_{m}\right)-T_{k}(u)\right) \eta_{j}\left(\left|u_{m}\right|\right) d x
\end{aligned}
$$

According to (27), (29); and $T_{k}\left(u_{m}\right) \rightarrow T_{k}(u)$ weakly in $\stackrel{\circ}{B}_{B}^{1}(\Omega), h_{j} \geq 0, \eta_{j}\left(\left|u_{m}\right|\right) \geq 0$, and $u_{m}\left(T_{k}\left(u_{m}\right)-\right.$ $\left.T_{k}(u)\right) \geq 0$ and $\exp (G( \pm \infty)) \leq \exp \left(\frac{\|l\|_{L^{1}(\mathbb{R})}}{\bar{a}}\right)$, we deduce that

$$
\sum_{i=1}^{N} \int_{\left\{\left|u_{m}\right| \leq j\right\}} a_{i}^{m}\left(x, u_{m}, \nabla u_{m}\right) \cdot \exp \left(G\left(\left|u_{m}\right|\right)\right)\left(\nabla T_{k}\left(u_{m}\right)-\nabla T_{k}(u)\right) \eta_{j}\left(\left|u_{m}\right|\right) d x \leq C(k, j, m) .
$$

Then,

$$
\begin{aligned}
\sum_{i=1}^{N} \int_{\Omega} & {\left[a_{i}\left(x, T_{k}\left(u_{m}\right), \nabla T_{k}\left(u_{m}\right)\right)-a_{i}\left(x, T_{k}\left(u_{m}\right), \nabla T_{k}(u)\right)\right] } \\
& \times \exp \left(G\left(\left|u_{m}\right|\right)\right)\left(\nabla T_{k}\left(u_{m}\right)-\nabla T_{k}(u)\right) \eta_{j}\left(\left|u_{m}\right|\right) d x \\
\leq & -\sum_{i=1}^{N} \int_{\Omega} a_{i}\left(x, T_{k}\left(u_{m}\right), \nabla T_{k}(u)\right) \cdot \exp \left(G\left(\left|u_{m}\right|\right)\right)\left|\nabla T_{k}\left(u_{m}\right)-\nabla T_{k}(u)\right| \eta_{j}\left(\left|u_{m}\right|\right) d x \\
& -\sum_{i=1}^{N} \int_{\left\{\left|u_{m}\right| \leq k\right\}} a_{i}\left(x, T_{k}\left(u_{m}\right), \nabla T_{k}(u)\right) \cdot \exp \left(G\left(\left|u_{m}\right|\right)\right) \nabla T_{k}(u) \eta_{j}\left(\left|u_{m}\right|\right) d x+C(k, j, m) .
\end{aligned}
$$

By Lebesgue dominated convergence theorem, we have $T_{k}\left(u_{m}\right) \longrightarrow T_{k}(u)$ strongly in $\mathfrak{W}_{B, l o c}^{1}(\Omega)$ and $\nabla T_{k}\left(u_{m}\right) \rightarrow \nabla T_{k}(u)$ weakly in $\mathfrak{W}_{B}^{1}(\Omega)$; then the terms on the right hand side of (50) go to zeros as $k, j, m$ tend to infinity, which gives

$$
\begin{aligned}
\sum_{i=1}^{N} \int_{\Omega}[ & \left.a_{i}\left(x, T_{k}\left(u_{m}\right), \nabla T_{k}\left(u_{m}\right)\right)-a_{i}\left(x, T_{k}\left(u_{m}\right), \nabla T_{k}(u)\right)\right] \\
& \times\left(\nabla T_{k}\left(u_{m}\right)-\nabla T_{k}(u)\right) \exp \left(G\left(\left|u_{m}\right|\right)\right) \eta_{j}\left(\left|u_{m}\right|\right) d x \longrightarrow 0 .
\end{aligned}
$$

By Proposition 4 and the diagonal process, we deduce for $k \longrightarrow \infty$ that

$$
B\left(\left|\nabla u_{m}\right|\right) \longrightarrow B(|\nabla u|) \text { in } L^{1}(\Omega) .
$$


Hence, we obtain for a subsequence

$$
\nabla u_{m} \longrightarrow \nabla u \text { a.e. in } \Omega \text {. }
$$

Step 5 . The equi-integrability of $b_{i}^{m}\left(x, u_{m}, \nabla u_{m}\right)$ :

In this step we will show that

$$
b_{i}^{m}\left(x, u_{m}, \nabla u_{m}\right) \longrightarrow b_{i}(x, u, \nabla u) .
$$

Therefore, it is enough to show that $b_{i}^{m}\left(x, u_{m}, \nabla u_{m}\right)$ is uniformly equi-integrable. We take the following test function

$$
v=u_{m}-\eta \exp \left(2 G\left(\left|u_{m}\right|\right)\right) \eta_{j}\left(\left|u_{m}\right|\right) T_{1}\left(u_{m}-T_{j}\left(u_{m}\right)\right) .
$$

We have

$$
\begin{aligned}
& \sum_{i=1}^{N} \int_{\Omega} a_{i}^{m}\left(x, u_{m}, \nabla u_{m}\right) \cdot \nabla\left(\exp \left(2 G\left(\left|u_{m}\right|\right)\right) \eta_{j}\left(\left|u_{m}\right|\right) T_{1}\left(u_{m}-T_{j}\left(u_{m}\right)\right)\right) d x \\
& \quad+\sum_{i=1}^{N} \int_{\Omega} b_{i}^{m}\left(x, u_{m}, \nabla u_{m}\right) \cdot \exp \left(2 G\left(\left|u_{m}\right|\right)\right) \eta_{j}\left(\left|u_{m}\right|\right) T_{1}\left(u_{m}-T_{j}\left(u_{m}\right)\right) d x \\
& \quad+\int_{\Omega} m \cdot T_{m}\left(u_{m}-\psi\right)^{-} \cdot s g_{\frac{1}{m}}\left(u_{m}\right) \cdot \exp \left(2 G\left(\left|u_{m}\right|\right)\right) \eta_{j}\left(\left|u_{m}\right|\right) T_{1}\left(u_{m}-T_{j}\left(u_{m}\right)\right) d x \\
& \quad \leq \int_{\Omega} f^{m}(x) \cdot \exp \left(2 G\left(\left|u_{m}\right|\right)\right) \eta_{j}\left(\left|u_{m}\right|\right) T_{1}\left(u_{m}-T_{j}\left(u_{m}\right)\right) d x
\end{aligned}
$$

then,

$$
\begin{aligned}
& \sum_{i=1}^{N} \int_{\Omega} a_{i}^{m}\left(x, u_{m}, \nabla u_{m}\right) \cdot \nabla u_{m} \cdot \frac{l\left(\left|u_{m}\right|\right)}{\bar{a}} \exp \left(2 G\left(\left|u_{m}\right|\right)\right) \eta_{j}\left(\left|u_{m}\right|\right) T_{1}\left(u_{m}-T_{j}\left(u_{m}\right)\right) d x \\
& \quad+\sum_{i=1}^{N} \int_{\Omega} a_{i}^{m}\left(x, u_{m}, \nabla u_{m}\right) \cdot \exp \left(2 G\left(\left|u_{m}\right|\right)\right) \nabla\left(\eta_{j}\left(\left|u_{m}\right|\right)\right) T_{1}\left(u_{m}-T_{j}\left(u_{m}\right)\right) d x \\
& \quad+\sum_{i=1}^{N} \int_{\Omega} a_{i}^{m}\left(x, u_{m}, \nabla u_{m}\right) \cdot \exp \left(2 G\left(\left|u_{m}\right|\right)\right) \eta_{j}\left(\left|u_{m}\right|\right) \nabla T_{1}\left(u_{m}-T_{j}\left(u_{m}\right)\right) d x \\
& \quad+\int_{\Omega} m \cdot T_{m}\left(u_{m}-\psi\right)^{-} \cdot \operatorname{sg}_{\frac{1}{m}}\left(u_{m}\right) \cdot \exp \left(2 G\left(\left|u_{m}\right|\right)\right) \eta_{j}\left(\left|u_{m}\right|\right) T_{1}\left(u_{m}-T_{j}\left(u_{m}\right)\right) d x \\
& \quad \leq \sum_{i=1}^{N} \int_{\Omega}\left|b_{i}^{m}\left(x, u_{m}, \nabla u_{m}\right)\right| \cdot \exp \left(2 G\left(\left|u_{m}\right|\right)\right) \eta_{j}\left(\left|u_{m}\right|\right) T_{1}\left(u_{m}-T_{j}\left(u_{m}\right)\right) d x \\
& \quad+\int_{\Omega} f^{m}(x) \cdot \exp \left(2 G\left(\left|u_{m}\right|\right)\right) \eta_{j}\left(\left|u_{m}\right|\right) T_{1}\left(u_{m}-T_{j}\left(u_{m}\right)\right) d x .
\end{aligned}
$$

By (2) and (4) we get

$$
\begin{aligned}
& \sum_{i=1}^{N} \int_{\Omega} a_{i}^{m}\left(x, u_{m}, \nabla u_{m}\right) \cdot \exp \left(2 G\left(\left|u_{m}\right|\right)\right) \nabla\left(\eta_{j}\left(\left|u_{m}\right|\right)\right) T_{1}\left(u_{m}-T_{j}\left(u_{m}\right)\right) d x \\
& \quad+\sum_{i=1}^{N} \int_{\Omega} a_{i}^{m}\left(x, u_{m}, \nabla u_{m}\right) \cdot \exp \left(2 G\left(\left|u_{m}\right|\right)\right) \eta_{j}\left(\left|u_{m}\right|\right) \nabla T_{1}\left(u_{m}-T_{j}\left(u_{m}\right)\right) d x \\
& \quad+\int_{\Omega} m \cdot T_{m}\left(u_{m}-\psi\right)^{-} \cdot g_{\frac{1}{m}}\left(u_{m}\right) \cdot \exp \left(2 G\left(\left|u_{m}\right|\right)\right) \eta_{j}\left(\left|u_{m}\right|\right) T_{1}\left(u_{m}-T_{j}\left(u_{m}\right)\right) d x \\
& \quad \leq \int_{\Omega}\left(h(x)+f^{m}(x)+\phi(x) \cdot \frac{l\left(\left|u_{m}\right|\right)}{\bar{a}}\right) \cdot \exp \left(2 G\left(\left|u_{m}\right|\right)\right) \eta_{j}\left(\left|u_{m}\right|\right) T_{1}\left(u_{m}-T_{j}\left(u_{m}\right)\right) d x
\end{aligned}
$$


we deduce that

$$
\begin{aligned}
& \sum_{i=1}^{N} \int_{\Omega} a_{i}^{m}\left(x, u_{m}, \nabla u_{m}\right) \cdot \exp \left(2 G\left(\left|u_{m}\right|\right)\right) \nabla\left(\eta_{j}\left(\left|u_{m}\right|\right)\right) T_{1}\left(u_{m}-T_{j}\left(u_{m}\right)\right) d x \\
& \quad+\sum_{i=1}^{N} \int_{\left\{j \leq\left|u_{m}\right| \leq j+1\right\}} a_{i}^{m}\left(x, u_{m}, \nabla u_{m}\right) \cdot \nabla u_{m} \exp \left(2 G\left(\left|u_{m}\right|\right)\right) \eta_{j}\left(\left|u_{m}\right|\right) d x \\
& \quad+\int_{\Omega} m \cdot T_{m}\left(u_{m}-\psi\right)^{-} \cdot g_{\frac{1}{m}}\left(u_{m}\right) \cdot \exp \left(2 G\left(\left|u_{m}\right|\right)\right) \eta_{j}\left(\left|u_{m}\right|\right) T_{1}\left(u_{m}-T_{j}\left(u_{m}\right)\right) d x \\
& \quad \leq \int_{\Omega}\left(h(x)+f^{m}(x)+\phi(x) \cdot \frac{l\left(\left|u_{m}\right|\right)}{\bar{a}}\right) \cdot \exp \left(2 G\left(\left|u_{m}\right|\right)\right) \eta_{j}\left(\left|u_{m}\right|\right) T_{1}\left(u_{m}-T_{j}\left(u_{m}\right)\right) d x
\end{aligned}
$$

Since $a_{i}^{m}\left(x, u_{m}, \nabla u_{m}\right)$ is bounded in $W_{B}^{1}(\Omega)$, and $\eta_{j}\left(\left|u_{m}\right|\right) \geq 0$ then by (27), (29) we obtain

$$
\begin{aligned}
& \sum_{i=1}^{N} \int_{\left\{j+1<\left|u_{m}\right|\right\}} B_{i}\left(\left|\nabla u_{m}\right|\right) d x \\
& \quad \leq \exp \left(2 \frac{|| l \|_{L^{1}(\mathbb{R})}}{\bar{a}}\right) \cdot \int_{\left\{j<\left|u_{m}\right|\right\}}\left[|C(x)|+\phi(x)+h(x)+f^{m}(x)+\phi(x) \cdot \frac{l\left(\left|u_{m}\right|\right)}{\bar{a}}\right] d x .
\end{aligned}
$$

Thus, $\forall \epsilon>0 \exists j(\epsilon)>0$ such that

$$
\sum_{i=1}^{N} \int_{\left\{j+1<\left|u_{m}\right|\right\}} B_{i}\left(\left|\nabla u_{m}\right|\right) d x \leq \frac{\epsilon}{2} \quad \forall j>j(\epsilon) .
$$

Let $\stackrel{\circ}{V}(\Omega(R))$ be an arbitrary bounded subset for $\Omega$; then, for any measurable set $E \subset \stackrel{\circ}{ }(\Omega(R))$ we have

$$
\begin{aligned}
& \sum_{i=1}^{N} \int_{E} B_{i}\left(\left|\nabla u_{m}\right|\right) d x \\
& \quad \leq \sum_{i=1}^{N} \int_{E} B_{i}\left(\left|\nabla T_{k}\left(u_{m}\right)\right|\right) d x+\sum_{i=1}^{N} \int_{\left\{j+1<\left|u_{m}\right|\right\}} B_{i}\left(\left|\nabla u_{m}\right|\right) d x .
\end{aligned}
$$

We conclude that $\forall E \subset \stackrel{\circ}{V}(\Omega(R))$ with meas $(E)<\beta(\epsilon)$, and $T_{k}\left(u_{m}\right) \longrightarrow T_{k}(u)$ in $\dot{W}_{B}^{1}(\Omega)$,

$$
\sum_{i=1}^{N} \int_{E} B_{i}\left(\left|\nabla T_{k}\left(u_{m}\right)\right|\right) d x \leq \frac{\epsilon}{2}
$$

Finally, by combining the last formulas we obtain

$$
\sum_{i=1}^{N} \int_{E} B_{i}\left(\left|\nabla u_{m}\right|\right) d x \leq \epsilon \quad \forall E \subset \stackrel{\circ}{V}(\Omega(R)) \text { such that meas }(E)<\beta(\epsilon),
$$

giving the assumed results.

Step 6. Passing to the limit:

Let $\varphi \in \stackrel{\mathrm{W}}{B}_{B}^{1}(\Omega) \cap L^{\infty}(\Omega)$; we take the following test function:

$$
v=u_{m}-\psi_{k} T_{k}\left(u_{m}-\varphi\right), \psi_{K} \in \mathcal{D}(\Omega),
$$


such that

$$
\psi_{k}(x)= \begin{cases}1 & \text { for } \Omega(R) \\ 0 & \text { for } \Omega(R+1) \backslash \Omega(R)\end{cases}
$$

and $\left|u_{m}\right|-|| \varphi \|_{\infty}<\left|u_{m}-\varphi\right| \leq j$. Then, by $\left\{\left|u_{m}-\varphi\right| \leq j\right\} \subset\left\{\left|u_{m}\right| \leq j+\|\varphi\|_{\infty}\right\}$ we obtain

$$
\begin{aligned}
& \sum_{i=1}^{N} \int_{\Omega(R+1)} a_{i}\left(x, T_{m}\left(u_{m}\right), \nabla u_{m}\right) \psi_{k} \nabla T_{k}\left(u_{m}-\varphi\right) d x \\
& \quad+\sum_{i=1}^{N} \int_{\Omega(R+1)} a_{i}\left(x, T_{m}\left(u_{m}\right), \nabla u_{m}\right) T_{k}\left(u_{m}-\varphi\right) \nabla \psi_{k} d x \\
& \quad+\sum_{i=1}^{N} \int_{\Omega(R+1)} b_{i}^{m}\left(x, u_{m}, \nabla u_{m}\right) \psi_{k} T_{k}\left(u_{m}-\varphi\right) d x \\
& \quad+\int_{\Omega(R+1)} m \cdot T_{m}\left(u_{m}-\psi\right)^{-} \cdot s g_{\frac{1}{m}}\left(u_{m}\right) \cdot \psi_{k} T_{k}\left(u_{m}-\varphi\right) d x \\
& \quad \leq \int_{\Omega(R+1)} f^{m}(x) \psi_{k} T_{k}\left(u_{m}-\varphi\right) d x,
\end{aligned}
$$

which implies that

$$
\begin{aligned}
& \sum_{i=1}^{N} \int_{\Omega(R+1)} a_{i}\left(x, T_{m}\left(u_{m}\right), \nabla u_{m}\right) \psi_{k} \nabla T_{k}\left(u_{m}-\varphi\right) d x \\
& \quad=\sum_{i=1}^{N} \int_{\Omega(R+1)} a_{i}\left(x, T_{j+\|\varphi\|_{\infty}}\left(u_{m}\right), \nabla T_{j+\|\varphi\|_{\infty}} u_{m}\right) \psi_{k} \nabla T_{k}\left(u_{m}-\varphi\right) d x \\
& \quad=\sum_{i=1}^{N} \int_{\Omega(R+1)}\left[a_{i}\left(x, T_{j+\|\varphi\|_{\infty}}\left(u_{m}\right), \nabla T_{j+\|\varphi\|_{\infty}}\left(u_{m}\right)\right)-a_{i}\left(x, T_{j+\|\varphi\|_{\infty}}\left(u_{m}\right), \nabla \varphi\right)\right] \\
& \quad \times \nabla T_{j+\|\varphi\|_{\infty}}\left(u_{m}-\varphi\right) \cdot \chi_{\left\{\left|u_{m}-\varphi\right|<j\right\}} d x \\
& \quad+\sum_{i=1}^{N} \int_{\Omega(R+1)} a_{i}\left(x, T_{j+\|\varphi\|_{\infty}}\left(u_{m}\right), \nabla \varphi\right) \nabla T_{j+\|\varphi\|_{\infty}}\left(u_{m}-\varphi\right) \cdot \chi_{\left\{\left|u_{m}-\varphi\right|<j\right\}} d x .
\end{aligned}
$$

By Fatou's Lemma we get

$$
\begin{aligned}
\lim _{m \rightarrow \infty} \inf & \sum_{i=1}^{N} \int_{\Omega(R+1)} a_{i}\left(x, T_{j+\|\varphi\|_{\infty}}\left(u_{m}\right), \nabla \varphi\right) \nabla T_{j+\|\varphi\|_{\infty}}\left(u_{m}-\varphi\right) \cdot \chi_{\left\{\left|u_{m}-\varphi\right|<j\right\}} d x \\
& =\sum_{i=1}^{N} \int_{\Omega(R+1)} a_{i}\left(x, T_{j+\|\varphi\|_{\infty}}(u), \nabla \varphi\right) \nabla T_{j+\|\varphi\|_{\infty}}(u-\varphi) \cdot \chi_{\{|u-\varphi|<j\}} d x
\end{aligned}
$$

and the fact that

$$
a_{i}\left(x, T_{j+\|\varphi\|_{\infty}}\left(u_{m}\right), \nabla T_{j+\|\varphi\|_{\infty}}\left(u_{m}\right)\right) \rightarrow a_{i}\left(x, T_{j+\|\varphi\|_{\infty}}(u), \nabla T_{j+\|\varphi\|_{\infty}}(u)\right)
$$

weakly in $\mathfrak{W}_{B}^{1}(\Omega)$. Additionally, since $\psi_{k} T_{k}\left(u_{m}-\varphi\right) \rightarrow \psi_{k} T_{k}(u-\varphi)$ weakly in $\mathfrak{W}_{B}^{1}(\Omega)$, and by (53) we obtain

$$
\sum_{i=1}^{N} \int_{\Omega(R+1)} b_{i}^{m}\left(x, u_{m}, \nabla u_{m}\right) \psi_{k} T_{k}\left(u_{m}-\varphi\right) d x \longrightarrow \sum_{i=1}^{N} \int_{\Omega(R+1)} b_{i}(x, u, \nabla u) \psi_{k} T_{k}(u-\varphi) d x,
$$


and

$$
\int_{\Omega(R+1)} f^{m}(x) \psi_{k} T_{k}\left(u_{m}-\varphi\right) d x \longrightarrow \int_{\Omega(R+1)} f(x) \psi_{k} T_{k}(u-\varphi) d x
$$

and

$$
\int_{\Omega(R+1)} m \cdot T_{m}\left(u_{m}-\psi\right)^{-} \cdot \operatorname{sg}_{\frac{1}{m}}\left(u_{m}\right) \cdot \psi_{k} T_{k}\left(u_{m}-\varphi\right) d x \longrightarrow \int_{\Omega(R+1)} m \cdot T_{m}(u-\psi)^{-} \cdot \operatorname{sg}_{\frac{1}{m}}(u) \cdot \psi_{k} T_{k}(u-\varphi) d x,
$$

so we get

$$
\begin{aligned}
& \sum_{i=1}^{N} \int_{\Omega(R+1)} a_{i}(x, u, \nabla u) \psi_{k} \nabla T_{k}(u-\varphi) d x+\sum_{i=1}^{N} \int_{\Omega(R+1)} a_{i}(x, u, \nabla u) T_{k}(u-\varphi) \nabla \psi_{k} d x \\
& \quad+\sum_{i=1}^{N} \int_{\Omega(R+1)} b_{i}(x, u, \nabla u) \psi_{k} T_{k}(u-\varphi) d x \\
& \quad+\int_{\Omega(R+1)} m \cdot T_{m}(u-\psi)^{-} \cdot s g_{\frac{1}{m}}(u) \cdot \psi_{k} T_{k}(u-\varphi) d x \\
& \quad \leq \int_{\Omega(R+1)} f(x) \psi_{k} T_{k}(u-\varphi) d x
\end{aligned}
$$

now passing to the limit to infinity in $k$, we obtain the entropy solution of the problem.

\section{Uniqueness of the Entropy Solution}

Theorem 3. Suppose that conditions (1)-(3) are true, and $b_{i}(x, u, \nabla u): \Omega \times \mathbb{R} \times \mathbb{R}^{N} \longrightarrow \mathbb{R}$ are strictly monotonic operators, at least for a broad class of lower order terms. Then, the problem $(\mathcal{P})$ has a unique solution.

Proof. Let $u$ and $\bar{u}$ belong to $K_{\psi} \cap L^{\infty}(\Omega)$ being two solutions of problem $(\mathcal{P})$ with $u \neq \bar{u}$. In accordance with Definition 6, we obtain

$$
\begin{aligned}
& \sum_{i=1}^{N} \int_{\Omega} a_{i}(x, u, \nabla u) \cdot \nabla(u-v) d x+\sum_{i=1}^{N} b_{i}(x, u, \nabla u) \cdot(u-v) d x \\
& \quad+\int_{\Omega} m \cdot T_{m}(u-\psi)^{-} \cdot \operatorname{sg}_{m}(u) \cdot(u-v) d x \\
& \quad \leq \int_{\Omega} f(x) \cdot(u-v) d x
\end{aligned}
$$

and

$$
\begin{aligned}
& \sum_{i=1}^{N} \int_{\Omega} a_{i}(x, \bar{u}, \nabla \bar{u}) \cdot \nabla(\bar{u}-v) d x+\sum_{i=1}^{N} b_{i}(x, \bar{u}, \nabla \bar{u}) \cdot(\bar{u}-v) d x \\
& \quad+\int_{\Omega} m \cdot T_{m}(\bar{u}-\psi)^{-} \cdot \operatorname{sg}_{m}(\bar{u}) \cdot(\bar{u}-v) d x \\
& \quad \leq \int_{\Omega} f(x) \cdot(\bar{u}-v) d x
\end{aligned}
$$

Denote $v=u-\mu(x)(u-\bar{u})(x)$ and $v=\bar{u}-\mu(x)(u-\bar{u})(x)$ with

$$
\mu(x)= \begin{cases}0 & \text { if } x \geq k, \\ k-\frac{|x|^{2}}{k} & \text { if }|x|<k, \\ 0 & \text { if } x \leq-k,\end{cases}
$$


as test functions in (60) and (61) respectively. Using (1), (27), (29) and the condition of a strictly monotonic for the operator $b_{i}(x, u, \nabla u)$, we substract the equations to obtain

$$
\sum_{i=1}^{N} \int_{\Omega}\left[a_{i}(x, u, \nabla u)-a_{i}(x, \bar{u}, \nabla \bar{u})\right] \cdot(u-\bar{u}) \nabla \mu(x) d x \leq 0,
$$

According to (6), we obtain

$$
\begin{aligned}
& \sum_{i=1}^{N} \int_{\Omega} \bar{B}_{i}\left(a_{i}(x, u, \nabla u)-a_{i}(x, \bar{u}, \nabla \bar{u})\right) d x+\sum_{i=1}^{N} \int_{\Omega} B_{i}((u-\bar{u}) \cdot \nabla \mu(x)) d x \\
& \quad \leq \sum_{i=1}^{N} \int_{\Omega} \bar{B}_{i}\left(a_{i}(x, u, \nabla u)-a_{i}(x, \bar{u}, \nabla \bar{u})\right) d x+2 \sum_{i=1}^{N} \int_{\Omega} B_{i}(u-\bar{u}) d x \\
& \quad \leq 0 .
\end{aligned}
$$

Since the $N$-functions $\bar{B}_{i}$ verified the same conditions and properties of $B_{i}$; then by (10), we have

$$
\begin{aligned}
& \sum_{i=1}^{N} \int_{\Omega} \bar{B}_{i}\left(a_{i}(x, u, \nabla u)-a_{i}(x, \bar{u}, \nabla \bar{u})\right) d x \\
& \quad \leq c \sum_{i=1}^{N} \int_{\Omega}\left|\bar{B}_{i}\left(a_{i}(x, u, \nabla u)\right)\right| d x-c \sum_{i=1}^{N} \int_{\Omega}\left|\bar{B}_{i}\left(a_{i}(x, \bar{u}, \nabla \bar{u})\right)\right| d x ;
\end{aligned}
$$

according to (3), we obtain

$$
\begin{aligned}
& \sum_{i=1}^{N} \int_{\Omega} \bar{B}_{i}\left(a_{i}(x, u, \nabla u)-a_{i}(x, \bar{u}, \nabla \bar{u})\right) d x \\
& \quad \leq \tilde{a} c \sum_{i=1}^{N} \int_{\Omega} B_{i}(\nabla(u-\bar{u})) d x \\
& \quad \leq \tilde{a} c\|B(u-\bar{u})\|_{1, \Omega} .
\end{aligned}
$$

Combined with (62) and (63) we get

$$
0 \leq(\tilde{a} c+2) \cdot\|B(u-\bar{u})\|_{1, \Omega} \leq 0 .
$$

Finally, $\|B(u-\bar{u})\|_{1, \Omega}=0$; therefore, $u=\bar{u}$ a.e. in $\Omega$.

Author Contributions: All authors performed all the steps of the ideas and proofs in this research. All authors have read and agreed to the published version of the manuscript.

Funding: This research received no external funding.

Conflicts of Interest: The authors declare no conflict of interest.

\section{Appendix A}

Let $S$ be the operator defined by

$$
\begin{aligned}
S(u)= & \sum_{i=1}^{N} \int_{\Omega} a_{i}^{m}(x, u, \nabla u) d x+\sum_{i=1}^{N} \int_{\Omega} b_{i}^{m}(x, u, \nabla u) d x+\int_{\Omega} m \cdot T_{m}(u-\psi)^{-} \cdot s g_{\frac{1}{m}}(u) d x \\
& -\int_{\Omega} f^{m}(x) d x,
\end{aligned}
$$


and for any $v \in \stackrel{\circ}{W}_{B}^{1}(\Omega), u \in \stackrel{\circ}{W}_{B, l o c}^{1}(\overline{\Omega(R)})$ we have

$$
\begin{aligned}
\langle S(u), v\rangle= & \sum_{i=1}^{N} \int_{\Omega(R)} a_{i}^{m}(x, u, \nabla u) \cdot \nabla v d x+\sum_{i=1}^{N} \int_{\Omega(R)} b_{i}^{m}(x, u, \nabla u) \cdot v d x \\
& +\int_{\Omega(R)} m \cdot T_{m}(u-\psi)^{-} \cdot \operatorname{sg}_{\frac{1}{m}}(u) \cdot v d x-\int_{\Omega(R)} f^{m}(x) \cdot v d x .
\end{aligned}
$$

In order to show the result of the Theorem 1, it is sufficient to show that operator $S$ is bounded, coercive and pseudo-monotonic.

Let us start by demonstrating that $S$ is bounded. Additionally, according to (A1), (15), (27) and (29) we obtain

$$
\begin{aligned}
|\langle S(u), v\rangle| \leq & 2\left\|a^{m}(x, u, \nabla u)\right\|\left\|_{\bar{B}, \Omega(R)} \cdot\right\| v\left\|_{\hat{W}_{B}^{1}(\Omega)}+2\right\| b^{m}(x, u, \nabla u)\|\|_{B, \Omega(R)} \cdot\|v\|_{\mathfrak{W}_{B}^{1}(\Omega)} \\
& -c_{0} \cdot\|v\|_{\mathfrak{W}_{B}^{1}(\Omega)} .
\end{aligned}
$$

or

$$
\left\|a^{m}(x, u, \nabla u)\right\|_{\bar{B}, \Omega(R)} \leq \sum_{i=1}^{N} \int_{\Omega(R)} \bar{B}_{i}\left(a_{i}^{m}(x, u, \nabla u)\right) d x+N
$$

and by diagonal process we obtain

$$
\left\|a^{m}(x, u, \nabla u)\right\|_{\bar{B}, \Omega(R)} \leq \bar{a}\|u\|_{\dot{W}_{B}^{1}(\Omega)}+\|\varphi\|_{1}+N
$$

and by (23) we deduce that

$$
b^{m}(x, u, \nabla u) \text { bounded in } L_{B}(\Omega)
$$

if $u$ with bounded support, and if support $u=\overline{\Omega(R)}$, then (A1) is bounded.

Next, we will move to proving that $S$ is coercive. By (27), (29) for any $u \in \mathfrak{W}_{B}^{1}(\Omega)$

$$
\langle S(u), u\rangle \geq \bar{a} \sum_{i=1}^{N} \int_{\Omega} B_{i}\left(\left|\frac{\partial u}{\partial x_{i}}\right|\right) d x-\int_{\Omega} \varphi(x) d x+\sum_{i=1}^{N} \int_{\Omega} b_{i}^{m}(x, u, \nabla u) \cdot u d x-\int_{\Omega} f^{m}(x) \cdot u d x,
$$

so

$$
\begin{aligned}
\frac{\langle S(u), u\rangle}{\|u\|_{\mathfrak{W}_{B}^{1}(\Omega)}^{1}} \geq & \frac{1}{\|u\|_{\mathfrak{W}_{B}^{1}(\Omega)}}\left[\bar{a} \sum_{i=1}^{N} \int_{\Omega} B_{i}\left(\left|\frac{\partial u}{\partial x_{i}}\right|\right) d x-c_{1}-c_{0}\right] \\
& +\frac{1}{\|u\|_{\mathfrak{W}_{B}^{1}(\Omega)}} \sum_{i=1}^{N} \int_{\Omega} b_{i}^{m}(x, u, \nabla u) \cdot u d x
\end{aligned}
$$

using (23), we obtain

$$
\frac{1}{\|u\|_{\mathfrak{W}_{B}^{1}(\Omega)}} \sum_{i=1}^{N} \int_{\Omega} b_{i}^{m}(x, u, \nabla u) \cdot u d x \geq-2 c(m) .
$$

Thus,

$$
\frac{\langle S(u), u\rangle}{\|u\|_{\mathfrak{W}_{B}^{1}(\Omega)}} \geq \frac{1}{\|u\|_{\mathfrak{W}_{B}^{1}(\Omega)}}\left[\bar{a} \sum_{i=1}^{N} \int_{\Omega} B_{i}\left(\left|\frac{\partial u}{\partial x_{i}}\right|\right) d x-c_{1}-c_{0}\right]-2 c(m)
$$

according to (22) we have for all $k>0, \exists \alpha_{0}>0$ such that

$$
b_{i}\left(\left|u_{x_{i}}\right|\right)>k b_{i}\left(\frac{\left|u_{x_{i}}\right|}{\left\|u_{x_{i}}\right\|_{B_{i}, \Omega}}\right) \quad i=1, \cdots, N \text {; }
$$


we take $\left\|u_{x_{i}}\right\|_{B_{i}, \Omega}>\alpha_{0}, i=1, \cdots, N$. Additionally, since $\Omega$ is unbounded domain, then we can assume that $\left\|u^{j}\right\|_{\mathscr{W}_{B}^{1}(\Omega)} \longrightarrow \infty$ as $j \longrightarrow \infty$. We suppose

$$
\left\|u_{x_{1}}^{j}\right\|_{B_{1}, \Omega}+\cdots+\left\|u_{x_{N}}^{j}\right\|_{B_{N}, \Omega} \geq N \alpha_{0}
$$

according to (9) we get

$$
\left|u^{j}\right| b\left(\left|u^{j}\right|\right)<c B\left(u^{j}\right),
$$

so,

$$
\begin{aligned}
\frac{\left\langle S\left(u^{j}\right), u^{j}\right\rangle}{\left\|u^{j}\right\|_{\mathfrak{W}_{B}^{1}(\Omega)}^{1}} & \geq \frac{\bar{a}}{N \alpha_{0}} \sum_{i=1}^{N} \int_{\Omega} B_{i}\left(\left|\frac{\partial u^{j}}{\partial x_{i}}\right|\right) d x-\frac{c_{2}}{N \alpha_{0}}-2 c(m) \\
& \geq \frac{\bar{a}}{c N\left\|u_{x_{i}}^{j}\right\|_{B_{i}}} \sum_{i=1}^{N} \int_{\Omega}\left|u_{x_{i}}^{j}\right| \cdot b_{i}\left(\left|u_{x_{i}}^{j}\right|\right) d x-\frac{c_{2}}{N \alpha_{0}}-2 c(m) \\
& \geq \frac{\bar{a} k}{c N} \sum_{i=1}^{N} \int_{\Omega} B_{i}\left(\frac{\left|u_{x_{i}}^{j}\right|}{\left\|u_{x_{i}}^{j}\right\|_{B_{i}, \Omega}}\right) d x-\frac{c_{2}}{N \alpha_{0}}-2 c(m),
\end{aligned}
$$

with $c_{2}=c_{0}+c_{1}$. Now, by the Luxemburg norm, we have

$$
\left\|u^{j}\right\|_{B}=\inf \left\{k>0 / \int_{\Omega} B\left(\frac{u^{j}(x)}{k}\right) d x \leq 1\right\}
$$

then

$$
\sum_{i=1}^{N} \int_{\Omega} B_{i}\left(\frac{\left|u_{x_{i}}^{j}\right|}{\left\|u_{x_{i}}^{j}\right\|_{B_{i}, \Omega}}\right) d x \geq \sum_{i=1}^{N}\left\|u_{x_{i}}^{j}\right\|_{B_{i}}
$$

Hence,

$$
\frac{\left\langle S\left(u^{j}\right), u^{j}\right\rangle}{\left\|u^{j}\right\|_{\mathfrak{W}_{B}^{1}(\Omega)}} \geq \frac{\bar{a} k}{c N}\left\|u^{j}\right\|_{\mathfrak{W}_{B}^{1}(\Omega)}-\frac{c_{2}}{N \alpha_{0}}-2 c(m) \longrightarrow \infty \text { as }\left\|u^{j}\right\|_{\mathfrak{W}_{B}^{1}(\Omega)} \longrightarrow \infty
$$

which gives the coercivity of the operator $S$.

Finally, we will end it by the demonstration of pseudo-monotonic of $S$. Following up this assumption, since the space $\stackrel{\circ}{B}_{B}^{1}(\Omega)$ is separable, then $\exists\left(u^{j}\right) \in C_{0}^{\infty}(\Omega)$ such that

$$
u^{j} \rightarrow u \text { in } \stackrel{\circ}{B}_{B}^{1}(\Omega),
$$

and

$$
S\left(u^{j}\right) \rightarrow y \text { in }\left(\stackrel{\circ}{W}_{B}^{1}(\Omega)\right)^{\prime} ;
$$

according to (A2), we have for all subsequences denoted again by $u^{j}$,

$$
\left\|u^{j}\right\|_{\aleph_{B}^{1}(\Omega)} \leq c_{2}, j \in \mathbb{N}
$$

$\left(u^{j}\right)_{j \in \mathbb{N}}$ is bounded in $\mathrm{W}_{B}^{1}(\Omega)$, and since $\mathrm{W}_{B}^{1}(\Omega)$ is continuously and compactly injected into $L_{B}(\Omega)$

$$
\begin{gathered}
u^{j} \rightarrow u \text { in } L_{B}(\Omega), \\
u^{j} \longrightarrow u \text { a.e. in } \Omega, j \in \mathbb{N},
\end{gathered}
$$

and according to (53), we have

$$
a_{i}^{m}\left(x, u^{j}, \nabla u^{j}\right) \longrightarrow a_{i}^{m}(x, u, \nabla u) \text { a.e. in } \Omega, j \in \mathbb{N}
$$


and

$$
b_{i}^{m}\left(x, u^{j}, \nabla u^{j}\right) \longrightarrow b_{i}^{m}(x, u, \nabla u) \text { a.e. in } \Omega, j \in \mathbb{N}
$$

and

$$
m \cdot T_{m}\left(u^{j}-\psi\right)^{-} \cdot \operatorname{sg}_{\frac{1}{m}}\left(u^{j}\right) \longrightarrow m \cdot T_{m}(u-\psi)^{-} \cdot \operatorname{sg}_{\frac{1}{m}}(u) \text { a.e. in } \Omega, j \in \mathbb{N}
$$

from (A2) and (A3), $\exists \tilde{a}^{m} \in L_{\bar{B}}(\Omega)$ such that

$$
a_{i}^{m}\left(x, u^{j}, \nabla u^{j}\right) \rightarrow \tilde{a}^{m}, j \in \mathbb{N}
$$

and $\exists \tilde{b}^{m} \in L_{B}(\Omega)$ such that

$$
b_{i}^{m}\left(x, u^{j}, \nabla u^{j}\right) \rightarrow \tilde{b}^{m}, j \in \mathbb{N} ;
$$

by (27) and (29) it is clear that for any $v \in \grave{W}_{B}^{1}(\Omega)$, we get

$$
\begin{aligned}
\langle y, v\rangle & =\lim _{j \rightarrow \infty} \sum_{i=1}^{N} \int_{\Omega} a_{i}^{m}\left(x, u^{j}, \nabla u^{j}\right) \cdot \nabla v d x+\lim _{j \rightarrow \infty} \sum_{i=1}^{N} \int_{\Omega} b_{i}^{m}\left(x, u^{j}, \nabla u^{j}\right) \cdot v d x \\
& =\int_{\Omega} \tilde{a}^{m} \cdot \nabla v d x+\int_{\Omega} \tilde{b}^{m} \cdot v d x
\end{aligned}
$$

whereof

$$
\begin{aligned}
\lim _{j \rightarrow \infty} \sup <S\left(u^{j}\right), u^{j}> & =\lim _{j \rightarrow \infty} \sup \left\{\sum_{i=1}^{N} \int_{\Omega} a_{i}^{m}\left(x, u^{j}, \nabla u^{j}\right) \nabla u^{j} d x\right. \\
& \left.+\lim _{j \rightarrow \infty} \sum_{i=1}^{N} \int_{\Omega} b_{i}^{m}\left(x, u^{j}, \nabla u^{j}\right) u^{j} d x\right\} \leq \int_{\Omega} \tilde{a}^{m} \nabla u^{j} d x+\int_{\Omega} \tilde{b}^{m} u^{j} d x,
\end{aligned}
$$

by (A5), we have

$$
\int_{\Omega} b^{m}\left(x, u^{j}, \nabla u^{j}\right) u^{j} d x \longrightarrow \int_{\Omega} \tilde{b}^{m} u d x
$$

consequently,

$$
\lim _{j \rightarrow \infty} \sup \sum_{i=1}^{N} \int_{\Omega} a_{i}^{m}\left(x, u^{j}, \nabla u^{j}\right) \nabla u^{j} d x \leq \int_{\Omega} \tilde{a}^{m} \nabla u^{j} d x .
$$

On the other hand, we have by the condition of monotony,

$$
\sum_{i=1}^{N}\left(a_{i}^{m}\left(x, u^{j}, \nabla u^{j}\right)-a_{i}^{m}\left(x, u^{j}, \nabla u\right)\right) \cdot \nabla\left(u^{j}-u\right) \geq 0
$$

which implies

$$
\sum_{i=1}^{N}\left(a_{i}\left(x, T_{m}\left(u^{j}\right), \nabla u^{j}\right)-a_{i}\left(x, T^{j}\left(u^{j}\right), \nabla u\right)\right) \cdot \nabla\left(u^{j}-u\right) \geq 0
$$

then;

$$
\sum_{i=1}^{N} a_{i}\left(x, T_{m}\left(u^{j}\right), \nabla u^{j}\right) \cdot \nabla u^{j} \geq \sum_{i=1}^{N} a_{i}\left(x, T_{m}\left(u^{j}\right), \nabla u\right) \cdot \nabla\left(u^{j}-u\right)+\sum_{i=1}^{N} a_{i}\left(x, T_{m}\left(u^{j}\right), \nabla u^{j}\right) \cdot \nabla u,
$$

and by Step 3, we get

$$
\sum_{i=1}^{N} a_{i}\left(x, T_{m}\left(u^{j}\right), \nabla u\right) \longrightarrow \sum_{i=1}^{N} a_{i}\left(x, T_{m}(u), \nabla u\right) \text { in } L_{\bar{B}}(\Omega) ;
$$


according to (A4), we obtain

$$
\lim _{j \rightarrow \infty} \inf \sum_{i=1}^{N} \int_{\Omega} a_{i}^{m}\left(x, u^{j}, \nabla u^{j}\right) \cdot \nabla u^{j} d x \geq \int_{\Omega} \tilde{a}^{m} \cdot \nabla u^{j} d x ;
$$

therefore, from (A9), we have

$$
\lim _{j \rightarrow \infty} \sum_{i=1}^{N} \int_{\Omega} a_{i}^{m}\left(x, u^{j}, \nabla u^{j}\right) \cdot \nabla u^{j} d x=\int_{\Omega} \tilde{a}^{m} \cdot \nabla u^{j} d x ;
$$

according to (A6), (A8) and (A11) we get

$$
<S\left(u^{j}\right), u^{j}>\longrightarrow<y, u>\text { as } j \rightarrow \infty .
$$

Hence, from (A12), and (53) we obtain

$$
\lim _{j \rightarrow \infty} \sum_{i=1}^{N} \int_{\Omega}\left(a_{i}^{m}\left(x, u^{j}, \nabla u^{j}\right)-a_{i}^{m}\left(x, u^{j}, \nabla u\right)\right) \cdot \nabla\left(u^{j}-u\right) d x=0 .
$$

By (A6) we can conclude that

$$
<y, u>=<S(u), u>\quad \forall u \in \grave{W}_{B}^{1}(\Omega) .
$$

\section{References}

1. Alber, H.D. Materials with Memory: Initial-Boundary Value Problems for Constitutive Equations with Internal Variables; Springer: New York, NY, USA, 2006.

2. Ball, J.M. Convexity conditions and existence theorems in nonlinear elasticity. Arch. Ration. Mech. Anal. 1976, 63, 337-403. [CrossRef]

3. Benkhira, E.H.; Essoufi, E.H.; Fakhar, R. On convergence of the penalty method for a static unilateral contact problem with nonlocal friction in electro-elasticity. Eur. J. Appl. Math. 2016, 27, 1-22. [CrossRef]

4. Chen, Y.; Levine, S.; Rao, M. Variable exponent, linear growth functionals in image restoration. SIAM J. Appl. Math. 2006, 66, 1383-1406. [CrossRef]

5. Bénilan, P.; Boccardo, L.; Gallouêt, T.; Gariepy, R.; Pierre, M.; Vázquez, J.L. An L11-theory of existence and uniqueness of solutions of nonlinear elliptic equations. Ann. Della Sc. Norm. Super. Pisa Cl. Sci. 1995, $22,241-273$.

6. Aberqi, A.; Bennouna, J.; Mekkour, M.; Redwane, H. Nonlinear parabolic inequalities with lower order terms. Appl. Anal. 2017, 96, 2102-2117. [CrossRef]

7. Benkirane, A.; Bennouna, J. Existence of solutions for nonlinear elliptic degenerate equations. Nonlinear Anal. Theory Methods Appl. 2003, 54, 9-37. [CrossRef]

8. Benslimane, O.; Aberqi, A.; Bennouna, J. Existence and Uniqueness of Weak Solution of $p(x)$-Laplacian in Sobolev Spaces with Variable Exponents in Complete Manifolds. arXiv 2020, arXiv:2006.04763.

9. Boureanu, M.M.; Udrea, C.; Udrea, D.N. Anisotropic problems with variable exponents and constant Dirichlet conditions. Electron. J. Differ. Equ. 2013, 2013, 1-13.

10. Aberqi, A.; Bennouna, J.; Elmassoudi, M.; Hammoumi, M. Existence and uniqueness of a renormalized solution of parabolic problems in Orlicz spaces. Monatshefte Math. 2019, 189, 195-219. [CrossRef]

11. Aharouch, L.; Bennouna, J. Existence and uniqueness of solutions of unilateral problems in Orlicz spaces. Nonlinear Anal. Theory Methods Appl. 2010, 72, 3553-3565. [CrossRef]

12. Demengel, F.; Hebey, E. On some nonlinear equations involving the $p$-Laplacian with critical Sobolev growth. Adv. Differ. Equ. 1998, 3, 533-574.

13. Evans, L.C. Weak Convergence Methods for Nonlinear Partial Differential Equations; American Mathematical Society: Providence, RI, USA, 1990.

14. Domanska, O.V. Boundary problems for elliptic systems with anisotropic nonlinearity. Visnik Nats. Univ. Lviv. Politekhnika Fiz. Mat. Nauki 2009, 660, 5-13. 
15. Bendahmane, M.; Karlsen, K.H. Nonlinear anisotropic elliptic and parabolic equations in $\mathbb{R}^{N}$ with advection and lower order terms and locally integrable data. Potential Anal. 2005, 22, 207-227. [CrossRef]

16. Bokalo, M.; Domanska, O. On well-posedness of boundary problems for elliptic equations in general anisotropic Lebesgue-Sobolev spaces. Mat. Stud. 2007, 28, 77-91.

17. Ayazoglu, R.; Akbulut, S.; Akkoyunlu, E. Uniform Boundedness of Kantorovich Operators in Variable Exponent Lebesgue Spaces. Filomat 2019, 33, 5755-5765. [CrossRef]

18. Cammaroto, F.; Vilasi, L. On a perturbed $\mathrm{p}(\mathrm{x})$-Laplacian problem in bounded and unbounded domain. J. Math. Anal. Appl. 2013, 402, 71-83. [CrossRef]

19. Ragusa, M.A.; Tachikawa, A. Regularity for minimizers for functionals of double phase with variable exponents. Adv. Nonlinear Anal. 2020, 9, 710-728. [CrossRef]

20. Korolev, A.G. Imbedding theorems for anisotropic Sobolev-Orlicz spaces. Vestnik Moskovskogo Universiteta. Seriya 1. Matematika. Mekhanika 1983, 38, 32-37.

21. Kozhevnikova, L.M. Existence of Entropic Solutions of an Elliptic Problem in Anisotropic Sobolev-Orlicz Spaces. J. Math. Sci. 2019, 241, 258-284. [CrossRef]

22. Kozhevnikova, L.M. On the entropy solution to an elliptic problem in anisotropic Sobolev-Orlicz spaces. Comput. Math. Math. Phys. 2017, 57, 434-452. [CrossRef]

23. Krasnosel'skii, M.A.; Rutickii, J.B. Convex Functions and Orlicz Spaces; Fizmatlit: Moscow, Russia, 1958.

24. Mihăilescu, M.; Moroşanu, G.; Rădulescu, V. Eigenvalue problems for anisotropic elliptic equations: An Orlicz-Sobolev space setting. Nonlinear Anal. Theory Methods Appl. 2010, 73, 3239-3253. [CrossRef]

25. Cianchi, A. A fully anisotropic Sobolev inequality. Pac. J. Math. 2000, 196, 283-294. [CrossRef]

26. Alberico, A.; Chlebicka, I.; Cianchi, A.; Zatorska-Goldstein, A. Fully anisotropic elliptic problems with minimally integrable data. Calc. Var. Partial. Differ. Equ. 2019, 58, 186. [CrossRef]

27. Boccardo, I.; Gallouët, T. Nonlinear elliptic equations with right hand side measures. Commun. Partial. Differ. Equ. 1992, 17, 189-258. [CrossRef]

28. Gossez, J.P. Nonlinear elliptic boundary value problems for equations with rapidly (or slowly) increasing coefficients. Trans. Am. Math. Soc. 1974, 190, 163-205. [CrossRef]

29. Lions, J.L. Quelques Méthodes de Résolution des Problemes aux Limites non Linéaires; Dunod: Paris, France, 1969.

(C) 2020 by the authors. Licensee MDPI, Basel, Switzerland. This article is an open access article distributed under the terms and conditions of the Creative Commons Attribution (CC BY) license (http:/ / creativecommons.org/licenses/by/4.0/). 